\title{
De la façon de nommer aux usages des plantes adventices des cultures en pays Jbala (nord du Maroc)
}

Relation des hommes et des femmes à l'espace agraire et à autrui

of the naming and uses of adventitious herbs of crops in Jbala region, Morocco.

Men and women's relationships to the agricultural land and to others

Louise Clochey et Yildiz Aumeeruddy-Thomas

\section{OpenEdition}

Édition électronique

URL : https://journals.openedition.org/ethnoecologie/3154

DOI : $10.4000 /$ ethnoecologie.3154

ISSN : 2267-2419

Éditeur

Laboratoire Éco-anthropologie

Référence électronique

Louise Clochey et Yildiz Aumeeruddy-Thomas, « De la façon de nommer aux usages des plantes adventices des cultures en pays Jbala (nord du Maroc) », Revue d'ethnoécologie [En ligne], Supplément 1 | 2017, mis en ligne le 17 octobre 2017, consulté le 19 novembre 2021. URL : http://

journals.openedition.org/ethnoecologie/3154; DOI : https://doi.org/10.4000/ethnoecologie.3154

Ce document a été généré automatiquement le 19 novembre 2021.

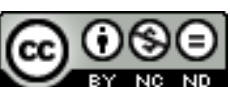

Revue d'ethnoécologie est mis à disposition selon les termes de la licence Creative Commons Attribution - Pas d'Utilisation Commerciale - Pas de Modification 4.0 International. 


\section{De la façon de nommer aux usages des plantes adventices des cultures en pays Jbala (nord du Maroc)}

Relation des hommes et des femmes à l'espace agraire et à autrui

of the naming and uses of adventitious herbs of crops in Jbala region, Morocco. Men and women's relationships to the agricultural land and to others

Louise Clochey et Yildiz Aumeeruddy-Thomas

\section{Introduction}

1 Les plantes adventices sont celles qui poussent sur les terres de culture transformées par les hommes, mais qui ne dépendent pas de ces derniers pour leur reproduction et leur survie (Colombel \& Tersis 1975). Certaines sont associées aux céréales et arrivent à maturité en même temps que celles-ci. Elles se nomment alors «messicoles", les plus emblématiques étant le coquelicot, le bleuet ...etc. Elles ont pour certaines voyagé avec les céréales (petit épeautre, blé, orge, seigle), domestiquées à l'est de la Méditerranée et ont été mises en culture ailleurs au cours des voyages des hommes. Ainsi, par exemple, Bouby (2000) analysant les restes archéobotaniques des céréales note que la gaude (Reseda luteola), plante tinctoriale de l'est méditerranéen est présente en Languedoc en tant que "mauvaise herbe» et émet l'hypothèse qu'elle ait pu voyager de l'est méditerranéen avec les céréales à l'âge du bronze.

2 Les plantes adventices sont aussi celles qui poussent sur les friches, ou aux alentours des maisons, en bordure des chemins ou dans certaines zones de pâtures. Les espèces adventices sont ainsi intrinsèquement associées à la niche socio-écologique crée par l'Homme. C'est grâce à la présence de ces flores adventices que les archéobotanistes identifient les premiers signes de l'agriculture. En effet, Willcox (2012) confirme la mise en culture des céréales car l'augmentation très significative des espèces adventices dans les restes archéobotaniques constitue un signal clair que les hommes ont 
augmenté de façon notable les surfaces cultivées. Certaines de ces espèces adventices peuvent être aussi des céréales comme le montre Bouby (2000) en Languedoc à l'âge du Bronze, où des espèces cultivées ailleurs tel le blé nu (Triticum aestivum / T. turgidum) et le millet commun (Panicum miliaceum) sont considérées comme des «mauvaises herbes ». Le ratio du nombre de graines trouvées par rapport au ratio de graines d'espèces cultivées est dans ce cas très faible. De même à cette période, des plantes telles que le lin (Linum usitatissimum) ou le pavot (Papaver somniferum) étaient alors pleinement domestiquées et cultivées, alors qu'elles sont des plantes adventices ou des rudérales aujourd'hui. Nous faisons références à ce statut changeant de certaines espèces adventices afin de signifier le caractère tout à fait relatif de cette notion de «mauvaise herbe ». En outre, elles participent à de multiples fonctions symboliques et matérielles au sein des univers domestiques, même si au cours de la modernisation agricole, elles furent dénommées "mauvaises herbes" ou "weeds» en anglais. Certains auteurs considèrent que toutes ces herbes sont potentiellement susceptibles d'être domestiquées et sont à l'origine de la plupart de nos graines et de nos légumes (Leonti et al. 2006).

3 En outre, il est largement reconnu dans de nombreuses régions que ces herbes sauvages, appelées «quelites» par exemple au Mexique (Lins Neto et al. 2014), ou «brèdes » en Afrique tropicale francophone (Garine-Wichatitsky 1997), à Madagascar et dans les îles de l'océan indien, tiennent une place considérable dans les cultures alimentaires locales, participant à diverses sauces et bouillons et apportant des éléments nutritifs essentiels. Spontanées, elles sont parfois favorisées et même, dans certains cas, semées.

$4 \mathrm{Au}$ Maroc, ces herbes participent à un plat nationalement reconnu, le Beqqula composé d'herbes sauvages cuites. Leur importance dans l'alimentation marocaine est connue depuis longtemps comme le souligne Rosenberger (1980) décrivant des pratiques alimentaires qui ont existé entre les $\mathrm{XV}^{\mathrm{e}}$ et $\mathrm{XVIII}^{\mathrm{e}}$ siècles au Maroc. Il rapporte ce texte pris de l'Ihiyâ $\hat{a}^{1}$ :

«Tous les jours les caravanes s'en allaient cueillir des herbes aux champs. Les gens cueillaient toutes les herbes qu'ils faisaient cuire ensemble et les mangeaient avec un peu de sel » ou alors « pendant quatre mois on a vécu d'herbes ».

5 Cueillies du nord au sud du Maroc, vendues sur les marchés, une multitude d'espèces servent à fabriquer au printemps des plats faits de mélanges d'herbes, cuites à la vapeur et assaisonnées d'huile d'olive et de quelques épices selon le goût. La Beqqula est abondamment consommé localement, de même que dans certains restaurants urbains.

Une des herbes sauvages de cueillette la plus connue est la mauve, qui porte le nom archétypique du plat Beqqula ou se nomme aussi Khobbiza; elle est aussi utilisée dans des tajines à base de plantes et elle est mangée au printemps avec du couscous d'orge et du petit lait. Nasif et Tangi (2013) ont effectué un premier état des lieux des herbes sauvages de cueillette à l'échelle du Maroc, sur la base des données bibliographiques existantes, issues d'une part des travaux de Bellakhdar $(1978,1997)$ et, d'autre part, d'une étude comparative à l'échelle méditerranéenne et d'une série d'enquêtes de terrain sur plusieurs années. Powell et al. (2014) ont compilé les données produites par Nasif et Tanji (2013) et y ajoutent de nouvelles données recueillies dans la région Nord du Maroc, dans la province de Taounate (dont dépend également notre site d'étude) et de deux sites du Centre et du Sud du Maroc. 
7 Cependant, ces travaux d'ethnobotanique n'explorent pas le terrain, les techniques directes ou indirectes qui favorisent la coexistence de ces espèces dans le paysage agricole avec les espèces cultivées, ou leur écologie spécifique en relation avec les différents espaces - biotopes reconnus et gérés localement. Par ailleurs, à ce jour, aucun travail n'explicite les savoirs et savoir-faire des groupes ruraux concernés, les représentations culturelles de ces plantes, leur utilisation et leur place dans le territoire, en lien avec les autres espèces qui les côtoient et les habitants. Seule la capacité des personnes enquêtées à les identifier et à les utiliser en témoigne, et reste garant de leur pérennité.

8 Ce papier est fondé sur des travaux menés à Ain Mediouna (commune de la Province de Taounate), habité par un groupe linguistique Jbala du nord du Maroc et tente d'expliciter ces différents aspects.

\section{Site d'étude, matériel et méthodes²}

9 Le corpus de données que nous présentons est issu d'une mission de terrain d'un mois réalisée au printemps 2014 dans la commune d'Ain Mediouna (Province de Taounate) par Louise Clochey qui a travaillé lors d'une immersion de trois semaines dans une famille de paysans (fellah) dans un des douars ${ }^{3}$ d'Ain Mediouna, Aouina Melha. YildizAumeeruddy-Thomas a accompagné cette mission pendant une période d'une semaine, pendant laquelle nous avons identifié les questions émergentes du terrain relatives au sujet d'étude, élaboré une première série d'enquêtes et avons mis au point les techniques de relevé ethnobotanique sur le terrain. Ces travaux avaient pour objectif de recueillir des informations sur les interactions des populations Jbala avec les plantes adventices des cultures céréalières et visaient à analyser les savoirs et savoir-faire, les usages et les représentations associées à ces dernières dans le cadre de l'espace agraire. Nous étions accompagnées par une linguiste, Dominique Caubet, professeur émérite d'arabe maghrébin à l'INALCO et chercheur attaché au Centre Jacques Berque de Rabat et une étudiante en thèse de linguistique, Fouzia el Ghazaz.

\section{Site principal : les environs de Ain Mediouna}

Ain Mediouna est une commune rurale de la province de Taounate et se situe dans le pré-Rif (Figure 1), sur les piémonts sud de la chaîne du Rif. 
Figure : 1 : Ain Mediouna, 90 km au nord-est de Fès, site principal d'étude

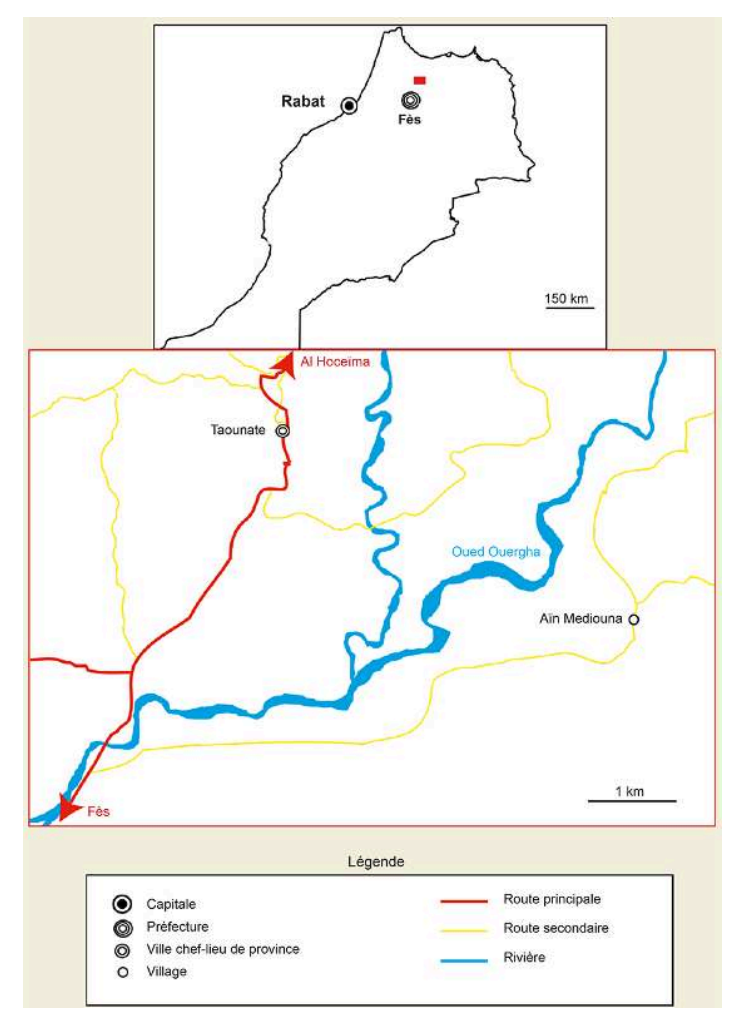

11 Ce site est situé à 312 mètres d'altitude. Sous climat méditerranéen, il bénéficie d'une pluviométrie annuelle de $601 \mathrm{~mm}$ en moyenne, condensée sur les mois d'hivers. La température varie entre des moyennes de 10 et $27,4^{\circ} \mathrm{C}$ en fonction des saisons. Les caractéristiques paysagères et d'exploitation des ressources sont telles que décrites pour l'ensemble du territoire rifain. Ain Mediouna est peuplée d'environ 16500 habitants appartenant à la tribu musulmane des Senhaja de Gheddo. Sur le plan économique, bien que le bourg comprenne de nombreuses infrastructures (école, lycée, hôpital, magasins) l'activité principale reste l'agriculture. Il semble que la production soit avant tout destinée à l'autoconsommation, surtout en ce qui concerne la production de céréales. Le système de vente agricole s'organise autour du marché local traditionnel, le souk (Troin 1975). Lieu de vie de Fouzia El Ghazaz, doctorante en ethnolinguistique de l'Université Sidi Mohamed Ben Abdellah de Fès, sa présence a permis l'introduction auprès de nombreuses personnes de son entourage. Au sein du village, l'étude a été réalisée dans deux sites directement chez l'habitant. Des enquêtes ont également eu lieu au souk hebdomadaire, et ce pendant trois semaines consécutives.

\section{Aouina Melha}

12 Aouina Melha est un petit hameau de Ain Mediouna, isolé sur une colline en dehors du village (douar), rassemblant trois familles de paysans (fellah). Le système agricole est conforme à toutes les caractéristiques du système agro-sylvo-pastoral rifain. Chaque famille possède ses propres terres, qu'elle exploite de manière rotative pour la culture de blé et d'orge, de fèves, de petits pois ou de pois chiche. On y trouve également de nombreuses zones de jachères qui sont des zones de pâturage pour les animaux (ovins, bovins). Pendant les trois semaines consécutives, l'étude a été réalisée via un 
hébergement au sein d'une de ces familles, vivant elle-même sur place depuis plus de 30 ans. Leur propre production (céréales, légumineuses, arbres fruitiers, jardin potager, produits laitiers et viande) est destinée avant tout à l'autoconsommation et subvient à une part importante de leurs besoins. Il en est de même pour l'ensemble des habitants du hameau. En saison, la vente de plantules (poivron, piment, aubergine) sur les souks de la région leur procure un complément financier.

\section{Tahriz}

Douar situé entre Ain Mediouna et Ain Aicha, Tahriz est le lieu de vie d'une famille de fellah et de leurs voisins, chez lesquels une enquête a été menée. Ils appartiennent également à la tribu des Sanhaja de Gheddo; selon les linguistes, aucune variation phonologique ne semble être observée dans leur parler par rapport au parler de Ain Mediouna.

\section{Switeyyin}

Switeyyin, hameau du pays Hyayna a également été visité dans le cadre de cette étude. Les Hyayna sont une confédération de tribus du pré-rif les plus importantes ayant une ancienne origine bédouine. Le pays comprend des collines marneuses, à sommet étroits, ayant entre 350 et $600 \mathrm{~m}$ d'altitude. Les Hyayna pratiquent depuis de nombreuses années la céréaliculture (orge, blé dur, petit épeautre) avant tout, dans les champs éparpillés sur les versants ou les fonds de vallée. Les caractéristiques climatiques du pays Hyayna sont similaires à celles de Ain Mediouna (températures, précipitations, couverture végétale etc.). La densité de population est également importante. Ce groupe parle l'arabe dialectal, avec des influences bédouines du fait de son histoire, les bédouins étant arrivés au Maroc à partir $\mathrm{du} \mathrm{xI}^{\mathrm{e}}$ siècle par le sud. Les Hyayna seraient arrivés au nord au xvI siècle sous l'influence des sultans Saadiens (voir Aumeeruddy-Thomas \& Caubet ce volume) alors que les Jbala sont issus de la première vague d'immigration arabe au $\mathrm{VII}^{\mathrm{e}}$ siècle qui se sont intégrés aux berbères Senhaja habitant ces régions. La famille visitée appartient donc à la tribu des Hyayna. Les Hyayna peuvent se marier avec les habitants de Ain Mediouna et une enquête a montré qu'ils possèdent certaines techniques en commun (Aumeeruddy-Thomas \& Caubet ce volume). Lors de notre séjour, nous avons rencontré une femme Hyayna mariée et vivant à Aouina Melha. Nous nous situons ainsi ici dans une situation de frontière entre deux groupes sociaux qui se distinguent par des parlers arabes et une histoire distinctes et qui maintiennent des différences linguistiques mais échangent sur différents plans (Caubet \& Aumeeruddy-Thomas, ce volume)

\section{Des paysages agraires complexes}

$15 \mathrm{Au}$ Maroc, et particulièrement dans les zones montagneuses du Rif, les systèmes traditionnels agro-sylvo-pastoraux sont toujours très présents. Cela entraine la présence d'une grande mosaïque de milieux cultivés, fragmentés, reliés à des zones de forêt. Le maintien des pratiques traditionnelles favorise une grande agrobiodiversité, d'espèces arborées, céréalières et de légumineuses (Hmimsa \& Ater 2008, Ater \& Hmimsa 2013, Hmimsa et al. 2012, Aumeeruddy-Thomas et al. 2017). Il est de surcroît rare que les habitants utilisent des pesticides et des intrants d'origine chimique, ce qui 
favorise la biodiversité spontanée. Selon Ater et Hmimsa (2013), l'absence de spécialisation et la pratique généralisée de la polyculture est hautement corrélée avec une grande richesse variétale des principales espèces cultivées. Les variétés rares locales et les variétés à diversité génétique élevée présentes dans le Rif sont des ressources de grand intérêt pour l'adaptation de ces agroécosystèmes dans un contexte écologique très hétérogène de montagne. Les céréales sont les cultures dominantes, du fait qu'elles sont fortement utilisées pour l'autoconsommation (alimentation humaine et animale). Dans le Rif on trouve de grandes cultures telles que les blés (Triticum turgidum et Triticum aestivum), le pois chiche (Cicer arietinum), la fève (Vicia faba), le petits pois (Pisum sativum). Elles présentent une abondance de variétés locales, parfois rares. Les cultures de sorgho (Sorghum bicolor) et de seigle (Secale cereale) sont de grand intérêt en termes d'agrodiversité. On observe également la persistance de cultures rares telles que celle du petit épeautre (Triticum monococum) ou des vesces (Vicia ervifilia et Vicia sativa). Cela souligne l'importance de ces agroécosystèmes en tant que refuge de l'agrodiversité et des pratiques associées.

16 Nous pouvions espérer que cette complexité paysagère et de l'agrobiodiversité locale soit associée à de nombreuses plantes adventices et il s'agissait de comprendre comment les paysans Jbala les appréhendent. À noter par ailleurs que la région est un hotspot de biodiversité à l'échelle internationale et abrite potentiellement une biodiversité importante (Médail \& Quézel 1999). Ces plantes adventices sont en outre connues pour s'exprimer au gré des rotations culturales et en interaction étroite avec les milieux hors champs (Petit et al. 2008). L'exploitation des terres de chaque douar se fait par unités destinées à des utilisations particulières. L'organisation de l'agriculture se fait de manière rotative, autour de cultures annuelles afin de rentabiliser l'atout climatique, en alternant de manière biennale entre céréales d'hiver et légumineuses de printemps. C'est donc l'ensemble de l'agroécosystème que nous avons considéré et c'est sur la flore sauvage ou spontanée dans sa globalité que nous avons effectué notre étude.

\section{Les enquêtes}

17 Les informations ont été récoltées au travers d'enquêtes ethnobotaniques ouvertes réalisées sur le terrain ou à la maison, complétées par des approches de Listes Libres (Free List). Le Free-List est une méthode mise au point dans le domaine de la psychologie cognitive dès le milieu du $\mathrm{xx}^{\mathrm{e}}$ siècle pour comprendre les mécanismes de catégorisation sémantique. La technique consiste à demander à des individus de faire la liste de l'ensemble des éléments appartenant à une catégorie donnée. Dans notre cas il s'agissait des plantes utilisées en Beqqula et des plantes utilisées pour l'alimentation de la vache (chaque maisonnée possède une à deux vaches) et d'autres animaux. Cette méthode permet d'établir une liste des taxons appartenant à un domaine donné et d'en définir les limites sémantiques. La fréquence avec laquelle les taxons sont cités ainsi que l'ordre dans lequel ils sont mentionnés donnent des indications sur la structure du domaine. Les taxons cités le plus fréquemment et le plus tôt dans les listes sont considérés être culturellement plus importants que les autres : en d'autres termes, ils sont considérés comme de meilleurs représentants de la catégorie étudiée (Pennec et al. 2012). Nous avons également effectué des marches le long de transects (transect walks) correspondant à diverses destinations et situations vécues quotidiennement par les habitants, lors desquelles non seulement nous repérions les plantes connues et identifiées par notre accompagnateur, les différents types d'espaces et les pratiques 
associées, mais nous profitions aussi des activités de nos hôtes pour mener des observations participantes et de courts entretiens de terrain souvent complétées à domicile à des moments opportuns. Toutes les plantes ont été mises en herbier et ont été identifiées au niveau des genres ou des espèces notamment avec l'aide d'un botaniste spécialiste de la flore du Maroc (Mr. Joël Mathez). La méthode des transect walks est couramment utilisée en ethnobotanique (Martin 1995) et permet de relever des données dans des situations où s'élaborent les pratiques et où les objets de l'enquête sont présents (espaces agraires, plantes, animaux domestiques). Cette technique diffère et peut donner des résultats différents des enquêtes à domicile, ou en dehors des situations vécues et qui portent sur des objets qui ne sont pas nécessairement présents pendant l'entretien.

Le système agro-sylvo-pastoral, pour l'autoconsommation à la fois des hommes et des animaux, est axé sur un principe d'autosubsistance. Si sa composition dans son ensemble est dominée par les céréales, les plantes sauvages qui y sont présentes ne font pas exception, et on observe un large panel de savoir et de savoir-faire gravitant autour de ces dernières. On observe ainsi des usages en termes d'alimentation humaine et animale, des usages médicinaux, des usages techniques, des usages ornementaux et divertissants.

Le printemps est favorable à la prolifération de la végétation et c'est à cette période qu'on trouve, juste avant la floraison, lorsque les plantes sont encore jeunes, une large gamme que l'on prépare en Beqqula. La flore spontanée est également ramassée par brassée pour fournir de l'alimentation à l'animal, aux jeunes veaux gardés à l'étable principalement. Nous avons effectué un suivi de la diversité des plantes fournies aux veaux et nous avons ouvert un échantillon de brassée et identifié avec nos hôtes les plantes qui les composent tout en les interrogeant sur les préférences des animaux et la façon dont ces brassées sont récoltées sur le terrain.

20 Enfin, afin de mettre en parallèle des informations écologiques et ethnobotaniques, des relevées d'abondance spécifique ont été réalisés pour identifier le cortège de plantes sauvages des zones présentant des faciès de pratiques culturales distinctes.

\section{Résultats}

\section{Beqqula}

21 L'exercice de free-list a permis de mettre en évidence une grande gamme de plantes adventices utilisées en Beqqula, variant selon les personnes interrogées. La plante archétype de la Beqqula est une espèce de mauve (possiblement plusieurs espèces selon Powell et al. 2014) (Malva sp.), appelée Khobbiza.

L'ensemble des plantes identifiées au cours de notre enquête (10 entretiens; enquête participante) sont réparties dans sept familles et 11 genres botaniques et ont des noms vernaculaires tous distincts qui sont dès lors des termes de base au sens de Friedberg (1986) qui qualifient chacune de ces plantes (Annexe 1) -. Nous avons observé et identifié 23 espèces qui sont récoltées dans la région de Ain Mediouna. Powell et al. (2014) ont identifié 29 espèces dans la commune voisine de Taounate.

23 Nous avons pu établir 11 listes libres ce qui est faible sur le plan statistique $(\mathrm{n}<30)$, mais cela permet néanmoins de calculer des fréquences. Le free listing (Figure 2) montre que 
Khobbiza (Malva sp.), est intégré dans les réponses à une fréquence de 90,9\%. Cette plante est donc largement la plus citée. Son indice de Sutrop $(0,313)$ indique qu'elle est rarement en tête de liste. Cet indice est proche de ceux d'une Asteraceae très connue localement nommée Guernina (Scolymus sp.) et Zarnij, une Asteraceae du même genre. Ces deux plantes ont des indices de Sutrop, respectivement de 0,22 et 0,23, et on les retrouve toutes deux dans $54,55 \%$ des réponses.

Figure 2 : Analyse de Free listing des espèces utilisées en Beqqula à Aouina Malha

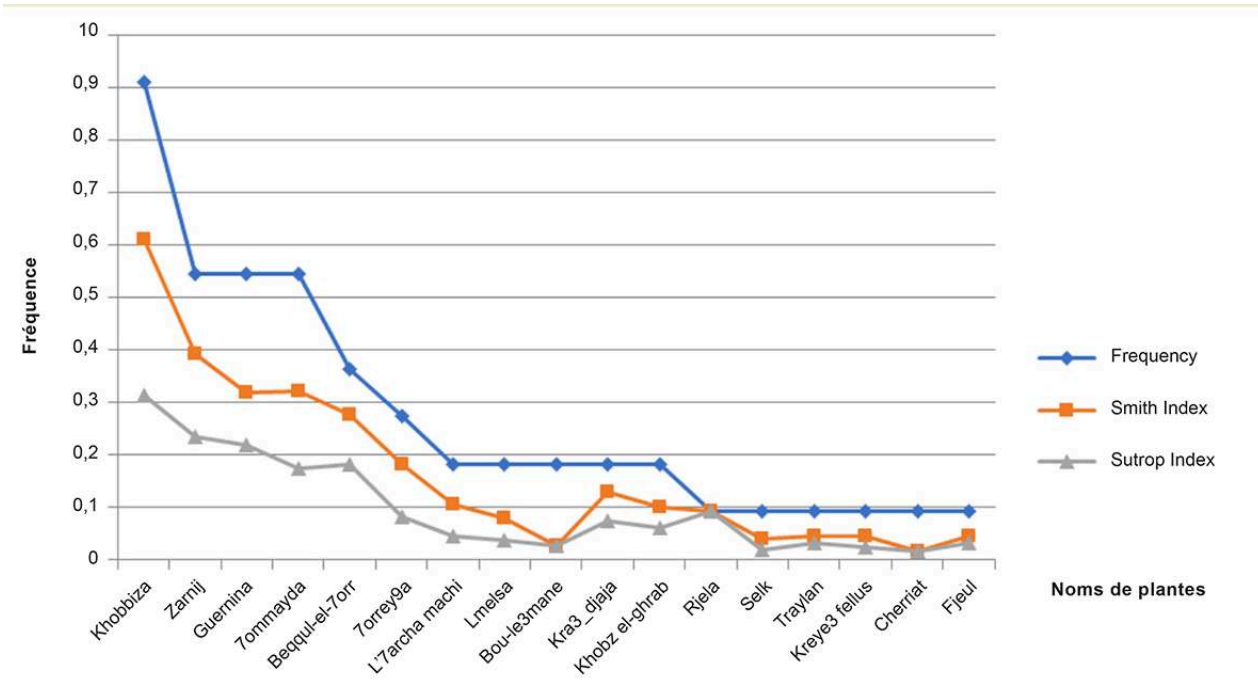

En observant la structure de ces résultats de Free listing en rapport avec les types de répondants, nous remarquons que seules les femmes citent Guernin/Guernina et Zarnij, la plupart du temps l'un à la suite de l'autre. Les hommes en revanche n'en citent qu'un, généralement Guernin. Ainsi l'un d'eux, vendeur au souk nous dit : «Cette plante a deux noms, Zarnij ou Guernin ${ }^{4}$ ». Et les femmes de renchérir : "c'est faux mais les hommes ne savent pas faire la différence ». 7ommayda, (Rumex sp.), est cité à 55,4 \% également, avec un indice de Sutrop égale à 0,17 . Les courbes des fréquences et indices de Sutrop chutent ensuite pour le reste des espèces. Les espèces citées appartiennent à une large gamme de famille : Malvaceae, Asteraceae, Polygonaceae, Brassicaceae.

\section{Plantes utilisées pour l'alimentation du bétail}

En ce qui concerne les plantes utilisées pour l'alimentation du bétail, l'exercice de Free listing réalisé pour les plantes fourragères a été relativement difficile à conduire, ou du moins les informateurs ont donné moins d'importance à cet exercice en comparaison de ceux réalisé pour la Beqqula. Complété par des transect walks et des méthodes d'observation participante, il a tout de même permis de mettre en évidence une large gamme d'espèces fourragères représentées par 50 espèces de plantes adventices, liées pour la plupart de manière exclusive à l'alimentation animale. Elles concernent tant l'ensemble du bétail que certaines familles particulières (bovin, caprin, équidé).

\section{Fréquence}

Chaque ballot fourni au veau gardé à l'étable fait en moyenne un poids de cinq kilos. La fréquence de chaque espèce, relevée juste avant que le ballot soit donné au veau, au 
cours des 7 jours de suivi, est très hétérogène (Figure 3). Avena sp. (Khortal), a une fréquence très élevée $(0,45)$, plus de quatre fois supérieure aux autres espèces. Cette espèce appartient à la famille des Poaceae. Trois autres espèces ressortent, avec une fréquence respective proche de 0,1: Rumex sp., (7ommayda), Trifolium sp., (Nefla) et Phalaris sp., (Zwan). Ils appartiennent respectivement à la famille des Polygonaceae, Fabaceae, Poaceae. L'ensemble des autres espèces sont moins représentées, elles appartiennent à des familles différentes. On constate une large dominance des Poaceae au sein des fourrages.

Figure 3 : Fréquences spécifiques des plantes de ballots fourragers

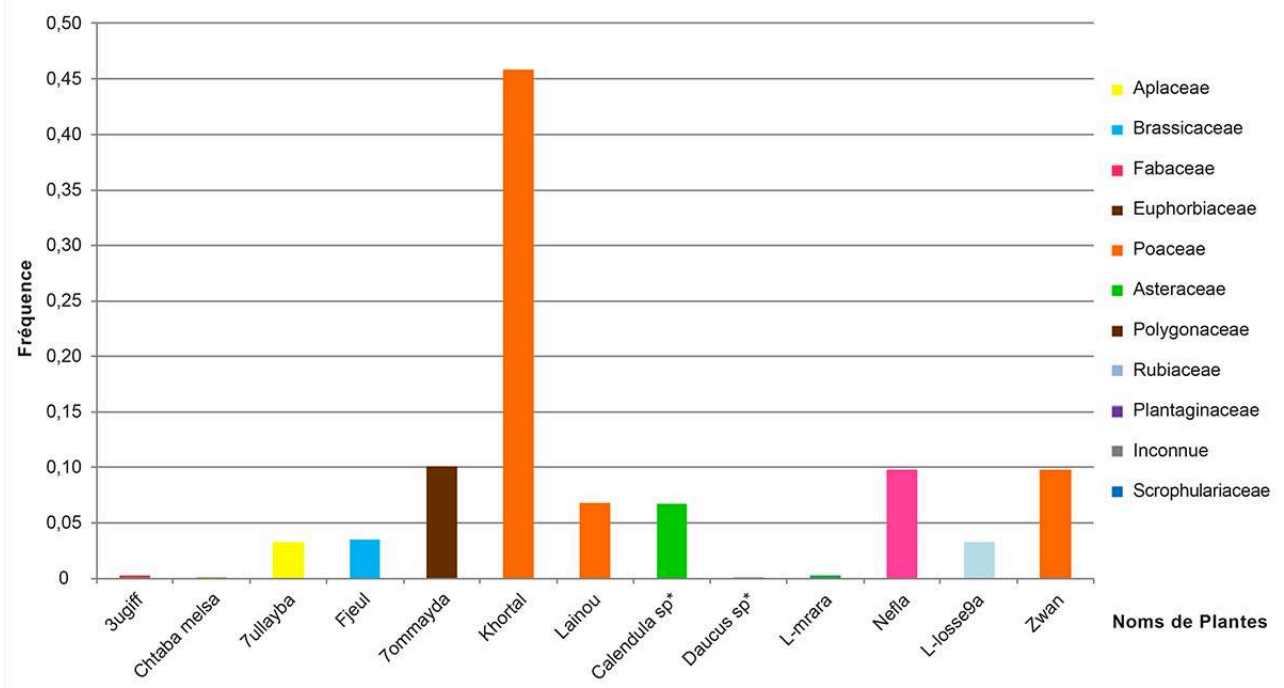

Si l'exercice de Free-List a présenté peu d'intérêt pour les informateurs, cela est dû fait qu'ils sont peu sélectifs dans leur choix de plantes fourragères. Lors du Free-List on affirme alors qu'hormis quelques espèces principales, toutes les plantes sont récoltées. Ce qui est confirmé avec cette observation du nourrissage : «- tu choisis ce que tu prends pour la vache? - Non je prends tout, c'est elle qui le fait » (un paysan d'Aouina Melha)

Ainsi, s'ils connaissent ce que la vache n'aime pas, et qu'ils ne prennent pas la peine de trier, c'est la biomasse qui est avant tout recherchée. Il convient tout de même de préciser que les connaissances sur ces plantes utilisées en alimentation animale sont néanmoins très élaborées, point sur lequel nous reviendrons par la suite. Cet ensemble témoigne d'une connaissance poussée par les populations locales des caractéristiques de chaque plante fourragère à travers l'animal et ses préférences alimentaires. Si les usages en eux-mêmes nous apportent des premières informations sur le lien des populations Jbala de Ain Mediouna aux plantes sauvages, nous devons les considérer également dans leur rapport au territoire.

\section{Une mosaïque agraire et des lieux de cueillette distincts}

Les lieux de cueillette sont une part incontournable du savoir et savoir-faire sur ces plantes, comme également montré à propos de la cueillette des asperges sauvages (Caubet \& Aumeeruddy-Thomas, ce volume). Au cours de nos enquêtes nous avons pu mettre en évidence une première information sur les différents noms attribués à la structuration de l'espace en différentes facettes écologiques interdépendantes. Les 
facettes écologiques telles que définies par la géographe Blanc-Pamard (1986) sont des unités paysagères identifiées et nommées distinctement les unes des autres, et désignées par un terme local ayant une signification et/ou renvoyant à des pratiques, à certaines phases ou modalités du travail paysan (Figure 4).

Figure 4 : Les différents types d'espaces de l'agroécosystème

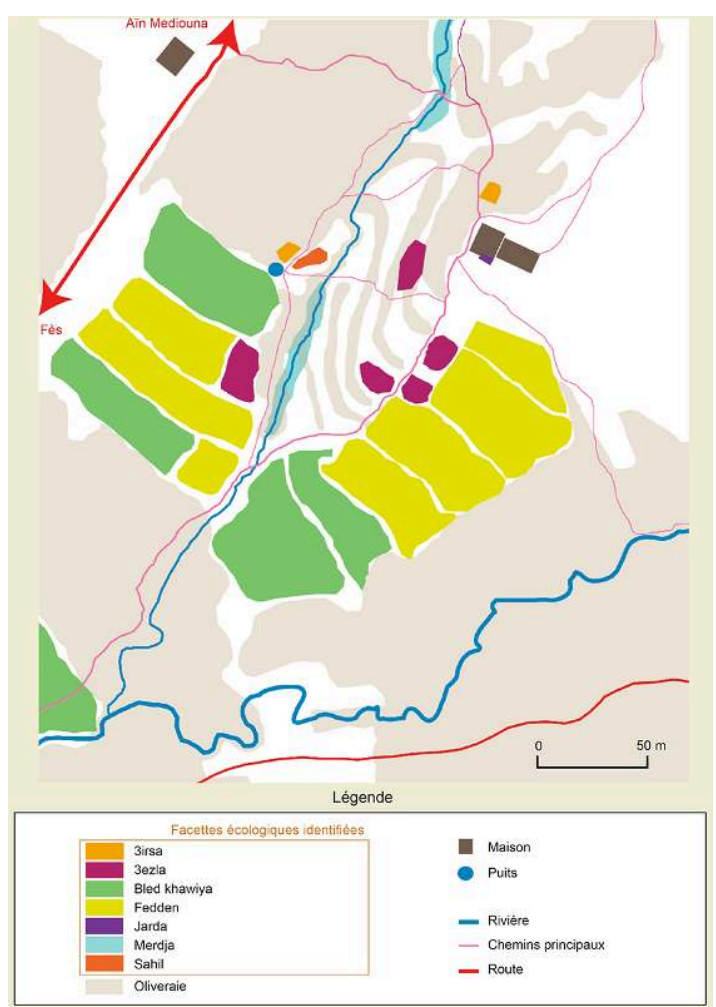

Les caractéristiques de ces différentes facettes écologiques sont données dans le tableau 1 ci-dessous.

\begin{tabular}{l|l}
\multicolumn{2}{l}{ Tableau 1: Caractéristiques des différentes facettes écologiques identifiées dans la figure 4 } \\
\hline 3irsa & jardin irrigué à plantations potagère mélangée. \\
\hline 3ezla & petit champ non irrigué à plantation unique \\
\hline Bled khawiya & terre vide (utilisée pour le pâturage) \\
\hline Fedden & champ (de céréales) \\
\hline Jarda & petit jardin en continuité avec la terrasse (plantes aromatiques, arbres et arbustes productifs) emprunt au français \\
\hline Merdja & zone verte en bordure de cours d'eau, parfois humide \\
\hline Sahil & petit jardin plat à plantation potagère mélangée
\end{tabular}

31 Cette mosaïque paysagère est parcourue de manière quotidienne (Figure 5). Cela se traduit par un grand nombre de chemins dans le territoire, permettant une circulation interne, et externe vers le village. Parmi les dynamiques quotidiennes, les vaches sont menées le matin et l'après-midi dans les grandes friches à l'extrémité des terres, arborées ou non. Il peut s'agir également des terres situées en contrebas des habitations puisque l'ensemble des friches disponibles sont utilisées pour le pâturage. Outre ces conduites en terre de pâturage, les vaches sont également menées quotidienne au puits pour l'abreuvage. L'ensemble de ces taches sont réalisées par les femmes. Des travaux aux champs sont également effectués aux heures fraiches de la journée, principalement par les femmes, mais également par les hommes en fonction de la nature : désherbage, plantation, irrigation des jardins potagers, récolte en champs 
ou en vergers, surveillance des bêtes... À tout cela s'ajoutent différents travaux épisodiques tels que les labours.

C'est dans ce contexte que s'effectuent les cueillettes: les plantes sauvages à feuilles de la Beqqula sont globalement réparties dans l'ensemble de l'espace agro-sylvo-pastoral: friches, humides ou non, jachère, bordure de champs ou chemins, champs de fève et de blé. La Beqqula est cependant faiblement associée au cœur même des cultures céréalières.

Une femme nous explique : "Elles poussent à côté des champs de blé ou de fève ou des deux. On les trouve n'importe où. Elles poussent quand il y a de la pluie." C'est ce qui est matérialisé en bleu sur la figure 5 , ci-dessous. Il convient de préciser que le lieu de récolte n'est jamais spécialement détaillé par les informateurs, même lorsqu'on leur pose la question. En effet, ils parcourent une importante partie de leur terre de manière quotidienne et n'ont pas besoin de le définir précisément. Ils insistent aussi sur le fait qu'elles poussent dans des lieux variables, en fonction des années : « elles poussent ici et là » (une femme âgée d'Aouina Melha).

Figure 5 : Des espaces de cueillettes distincts

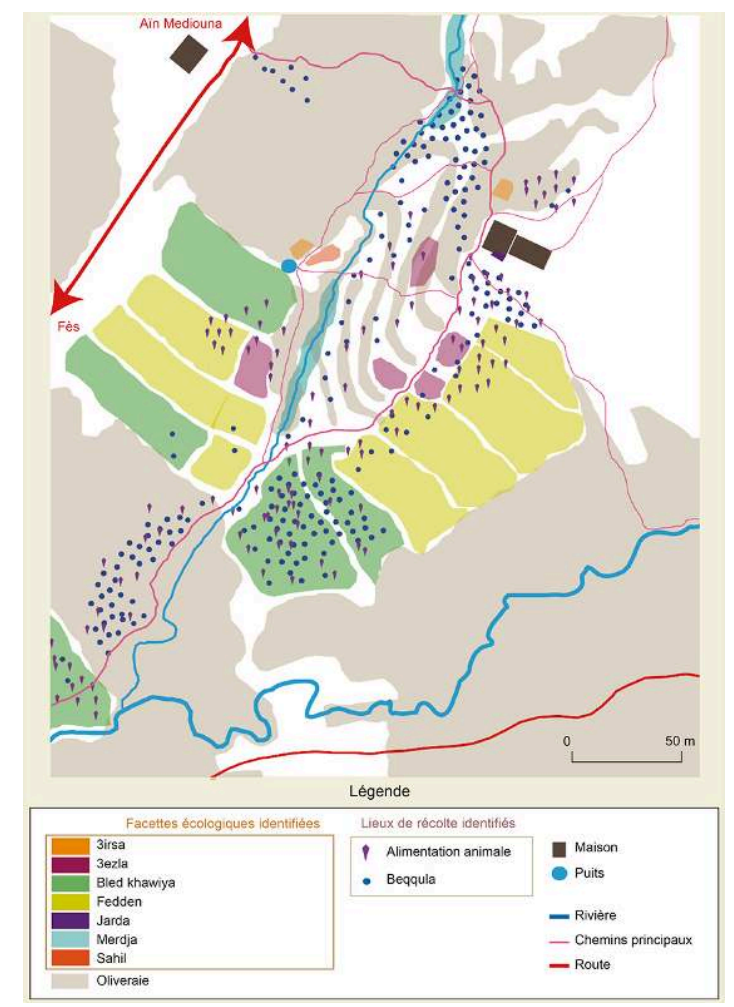

Et, si l'ensemble est parcouru avant tout par les femmes comme déjà évoqué, ce sont aussi les femmes qui effectuent ces travaux de cueillette. "C'est moi qui ramasse et ma fille qui prépare " témoigne une vielle dame du souk. "Ma femme ramasse ", complète plus tard un vendeur.

En ce qui concerne l'alimentation animale, l'homme est plus souvent mobilisé. C'est lui qui gère généralement les champs cultivés où les plantes sont largement présentes. Poaceae largement majoritaire en champs de blé et d'orge, l'avoine ou khortal (Avena sp.) est favorisée voir plantée au sein même de ces cultures (Figure 5, zones violettes). Lorsque nous demandons à un vendeur du souk : «Tu utilises les plantes qui poussent dans 
le blé? » il répond : "oui pour mes vaches». On trouve également ces plantes en champs de fève, ou dans les jachères, zones de prélèvement garantissant une forte biomasse d'adventices. Enfin, outre ces zones de prélèvement, un élément essentiel à la réalisation de la cueillette repose dans la capacité d'identification des plantes. Si nous avons évoqué le fait que ce sont les femmes qui parcourent largement et principalement le territoire agro-sylvo-pastoral pour les activités quotidiennes, d'élevage entre autres, ce sont elles également qui possèdent les connaissances sur les plantes sauvages.

\section{Les savoirs botaniques}

\section{Savoirs des femmes, des hommes et des enfants}

Les transects walk effectués dans les trois sites d'études et les relevés de diversité en champs à Aouina Malha ont permis de recueillir 119 noms locaux de plantes ${ }^{5}$. La question posée aux interlocuteurs rencontrés qui ont bien voulu se prêter à l'exercice d'identification ( $\mathrm{n}=24$; figure 6) était la suivante : "Vous connaissez le nom des plantes qu'il y a dans les champs? " Qu'ils soient paysans d'âge moyen, citadins, fils de paysan, paysannes d'âge mur "Oui je les connais tous... » est la réponse que nous avons obtenue dans beaucoup de cas. Quelques mises en pratique ont permis de vérifier cela. Nous avons utilisé 19 espèces (Annexe 1, en gras dans le tableau) assez fréquentes que nous avons présentées à nos interlocuteurs afin de vérifier lesquels ils reconnaissaient.

Figure 6 : Représentation visuelle des connaissances relatives en fonction du lieu, du sexe et de l'âge de l'informateur

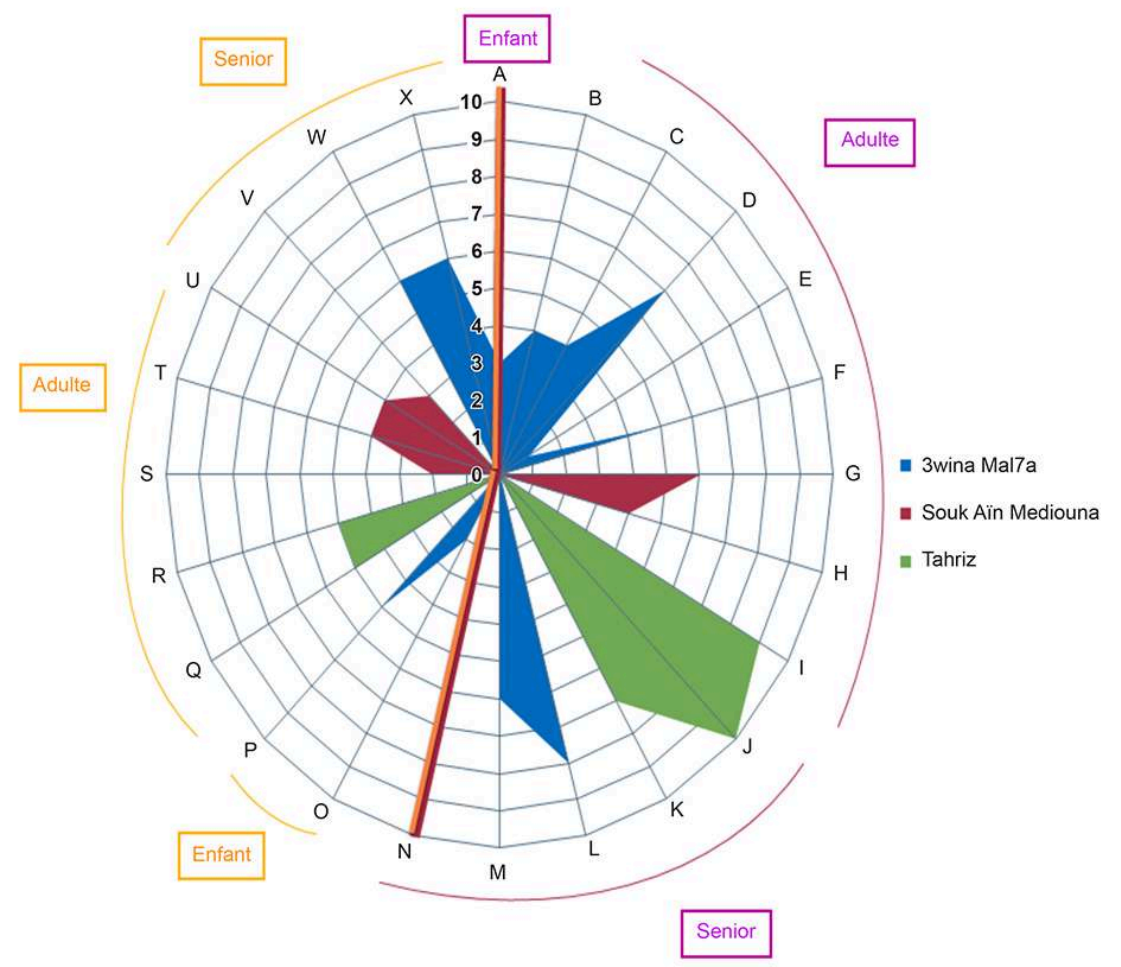

La figure 6 ci-dessus représente l'ensemble des notes attribuées à chaque informateur lors de l'exercice d'indentification. Chaque lettre représente un informateur. Plus la 
forme géométrique représentant la note de l'individu est proche de la périphérie du spider chart plus la note est élevée. Dans un premier temps, on observe que les formes géographiques liées aux femmes (en rose) représentent une aire plus importante que celles des hommes (en orange) ce qui dénoterait un savoir plus étendu des femmes en matière d'indentification. L'âge semble également avoir un impact. En suivant le même principe d'interprétation du graphe que pour le genre, on observe que les notes des femmes âgées ( $>50)$ sont largement supérieures, atteignant jusqu'à 10/10 comparée à celles des femmes adultes (entre 15 et 50 ans.), atteignant un maximum de 7/10. La seule enfant qui a été interrogée a une note relativement faible (3/10). Pour les hommes, les notes en fonction de l'âge sont plus homogènes. Les individus d'âge mur $(>50)$ ont des résultats allant jusqu'à $6 / 10$ et donc légèrement supérieurs à ceux des hommes d'âge moyen (au maximum 5/10). Un des garçons obtient une note de 5/10.

L'ensemble de ces observations est confirmé par le spider chart des notes en fonction de la combinaison de l'âge et du sexe (Figure 7) : les groupes d'individus qui obtiennent les meilleures notes sont classés dans l'ordre suivant: Femme âgée > Femme adulte=Homme âgé $>$ Homme adulte ( $>$ Garçon $>$ Fille). Notons cependant que concernant les enfants, le faible nombre ne peut nous donner une tendance représentative. La question de la transmission des connaissances a été abordée lors de cet exercice. Nombreux affirment qu'ils « habitent là depuis tout petit, j'ai appris comme $c ̧ a »$. Les parents semblent être impliqués, avant tout pour les femmes. Ils sont cependant moins cités que le cercle social, incluant famille et amis. C'était le cas, par exemple, pour ce paysan de Tahriz: «j'ai entendu les noms de gens plus grands que moi. Par exemple Le7yat 1-3atros, je le mangeais dans les champs avec mon cousin et c'est lui qui m'a appris son nom ».

Figure 7 : Notes moyennes en fonction de l'âge et du sexe

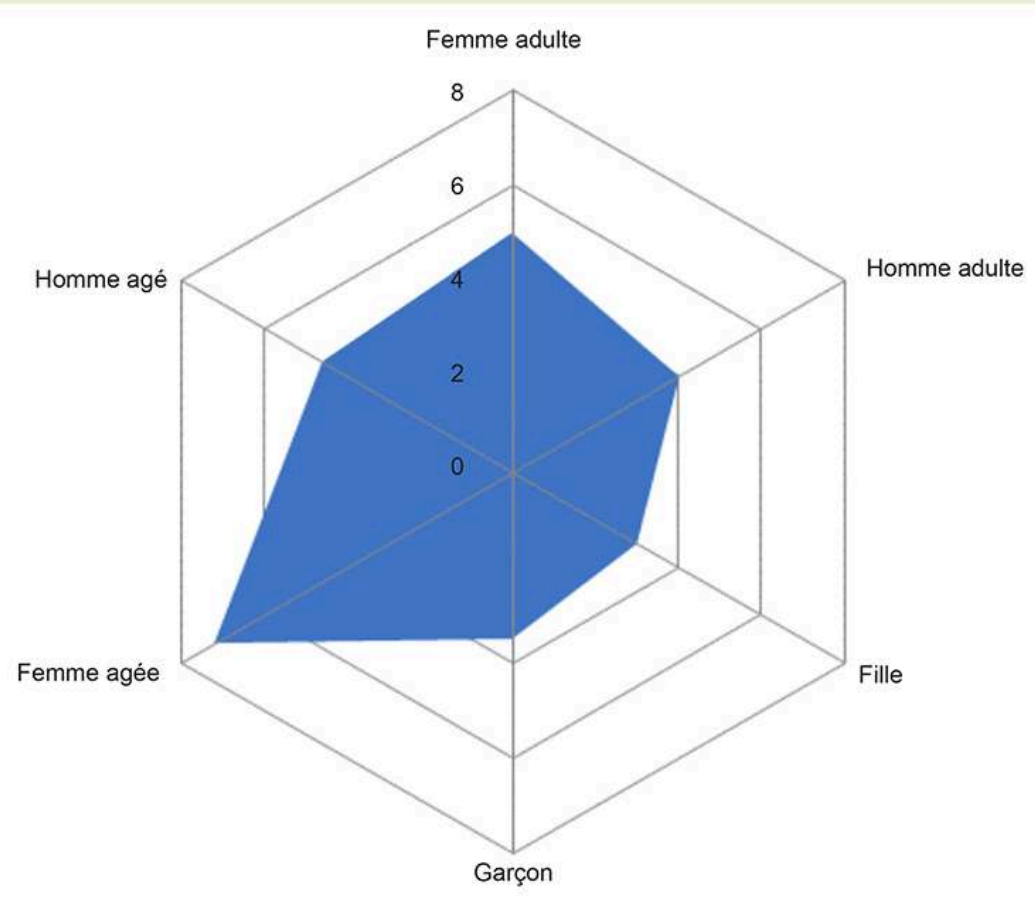

On observe une différence entre les notes en fonction des lieux. Les exercices réalisés in situ, avec les plantes du champ des individus, regroupe l'ensemble des meilleures notes 
obtenues. À l'inverse, les personnes du souk ont globalement des notes très moyennes par rapport au reste des individus de la catégorie "Genre » ou « Âge » à laquelle elles appartiennent.

Ces résultats sont appuyés par des enquêtes et observations de terrain. Lorsqu'il a été demandé d'identifier les plantes ex situ, lors de la mise en herbier à domicile, même les personnes supposées détenir le plus de connaissances botaniques faisaient des erreurs; par exemple, une des personnes référentes a confondu une Scrophulariaceae (non déterminée, Linaria sp., $\mathrm{n}^{\circ}$ herbier LCYAT $94^{6}$ ) avec une Orobanchaceae (Chowwwel Lakhrof), qu'elle reconnait pourtant parfaitement in situ.

\section{Différenciation des savoirs selon les usages des plantes}

41 La figure 8 ci-dessous rassemble l'ensemble des plantes ayant été utilisées pour cet exercice d'identification, réparties dans différentes classes en fonction de leur principale utilisation. Elle montre que les espèces pour lesquelles les notes moyennes sont les plus élevées, entre 4,7 et 7,5, sont utilisées pour l'alimentation animale. Le groupe de plantes utilisées pour l'alimentation humaine sont liés à des individus ayant une note moyenne relativement importante, de 4 ou 5. Il en est de même pour Chowwal L-khrof, Orobanche sp., bien connu des agriculteurs pour «tuer toutes les fèves sur $1 \mathrm{~m}^{2} \mathrm{de}$ champ ", et pour cause, il s'agit d'une plante non-chlorophyllienne parasite. On remarque de plus que les individus pour lesquels aucune utilisation n'a été reportée possèdent des notes plus hétérogènes, basses voir très basses $(-1,5)$. Par ailleurs, si l'on s'intéresse aux familles des plantes de cet exercice, trois d'entre elles ne possèdent que des notes élevées. Les Poaceae ont une moyenne de 7 et 7,5, les Fabaceae de 4,7 et 6, les Brassicaceae de 4,7 et 6 . Le nombre est très variable pour les Asteraceae

Figure 8 : Note moyenne associée à chaque plante en relation avec son usage

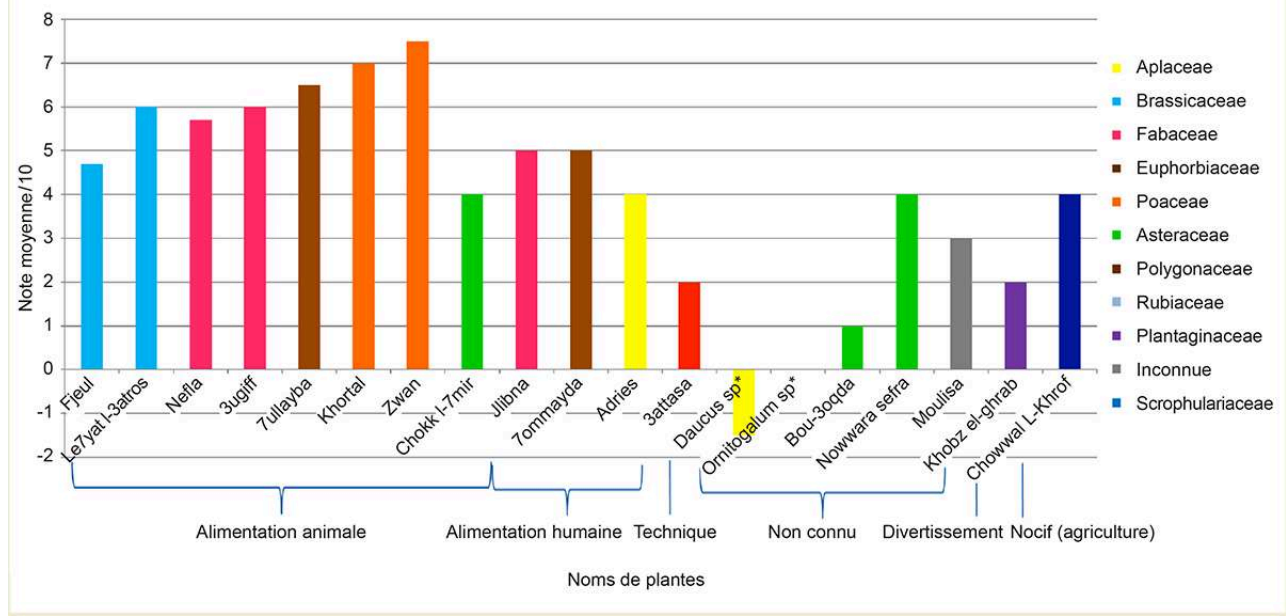

\section{Les noms en disent long}

Si le nom semble être l'outil principal de différenciation des plantes, précisons d'abord que cela n'est pas toujours le cas. Des plantes d'une même famille peuvent être simplement regroupées sous le même nom, par manque de nécessité de les différencier du fait d'un non-usage ou parce qu'on les connait parfaitement, et dans ce cas, il n'est 
pas besoin de les différencier par les noms. C'est le cas de Lewwaya (Campanula sp.) regroupant plusieurs espèces, simplement citée comme décorative. Un autre exemple est celui de Khobbiza (Malva sp.), correspondant à deux espèces de mauves, une utilisée pour la Beqqula, l'autre pour le bétail. La population locale sait les différencier sans avoir pour autant besoin de leur attribuer un nom différent à chacune.

La manière de nommer reste tout de même d'une extrême richesse et est largement révélatrice de la nature des liens que les gens établissent avec ces plantes, ainsi que des représentations qu'ils en ont. Étudier leur manière de nommer a permis de compléter et déceler les connaissances intimes et raffinées qu'ils ont des plantes. Les noms servent tant pour l'identification que pour les usages, mais renvoie aussi à des univers cognitifs qui en disent long sur l'imaginaire de ceux qui les utilisent. Ces manières de nommer reflètent la manière dont la société interprète, perçoit et utilise cet élément de son environnement, et de la relation qui l'unit à ce dernier.

Sur l'ensemble des noms de plantes recueillis, $31 \%^{7}$ seulement sont, à notre connaissance à l'issue de ce travail, motivés et évoquent en outre des univers très variés (Tableau 2).

\begin{tabular}{l|l}
\hline \multicolumn{2}{l}{ Tableau 2: Exemples de noms motivés } \\
\hline 9ossat l'7ayya & Couronne de la vipère \\
\hline Gouriba d-s-S3ay & Comme la jarre \\
\hline Fowwa7a/Bou-3aej n7al & Que les abeilles ne touchent pas \\
\hline Le7yat l-3atros & Barbe de bouc
\end{tabular}

La motivation utilisée en linguistique (Colombel \& Tersis 2002) permet en ethnobotanique de comprendre comment les hommes se représentent le monde végétal, quels aspects retiennent leur attention, à quel domaine de la vie ou à quel autre être vivant ces plantes sont associées.

Les plantes dont les noms n'évoquent rien ont possiblement une origine lointaine et ont pu perdre aujourd'hui leur sens si ce n'est celui de nommer un objet. Dans une société ou le berbère a constitué la langue de base depuis des millénaires et ou l'arabe est arrivé relativement récemment, on peut imaginer que des transformations, ou des réinterprétations des termes ne permettent pas aujourd'hui de comprendre leur étymologie. Parmi ces plantes dont le nom est motivé, plus de $32 \%$ font référence à des analogies de l'apparence de la plante avec un animal, 27\% à des caractéristiques propres à la plante elle-même (Tableaux 3 et 4 ).

\begin{tabular}{|c|c|}
\hline Matecha d'I7aya & La tomate de la vipère \\
\hline 2arn el Kebch & Les cornes du mouton \\
\hline Rjiyyel fellus & Petit pied du poussin \\
\hline Chowwal L-Khrof & Queue du mouton \\
\hline Z-ztema de ddib & Empreinte du loup \\
\hline \multicolumn{2}{|c|}{ Tableau 4: Exemples de plantes dont le nom se réfère aux caractéristiques physico-chimiques de la plar } \\
\hline Bou-3oqda & Celle avec un nœud \\
\hline L-losse9a & Collante \\
\hline Nowwara sefra & Fleur jaune \\
\hline 7ullayba & Laiteuse \\
\hline Mkhinza & Puante \\
\hline
\end{tabular}


Un très faible pourcentage évoque une comparaison physique avec des outils domestiques ou une référence personnelle de ressemblance à un individu, un aspect religieux ou l'impact de la plante sur les Hommes (Tableaux 5, 6 et 7).

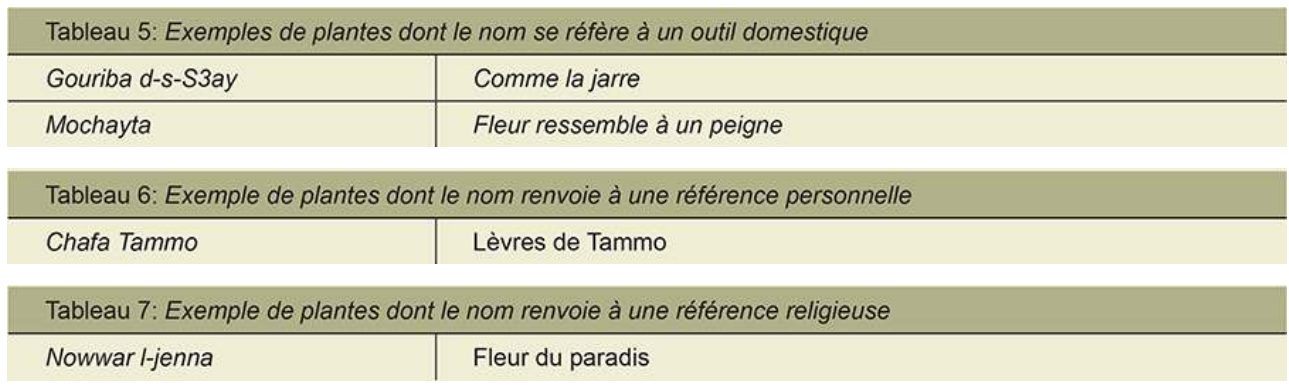

Enfin, outre ces analogies physiques relativement directes, $27 \%$ font directement référence à la fonction de ces plantes : fonction médicinale, technique, d'alimentation animale, etc., des termes qui peuvent être un biais pour transmettre des paroles signifiantes en lien avec l'usage de ces plantes (Tableau 8).

\begin{tabular}{l|l}
\multicolumn{2}{|l|}{ Tableau 8: Exemples de noms faisant référence à la fonction des plantes } \\
\hline Mrinsla/chettaba melsa & Balai lisse \\
\hline Chokk l-7mir & Les épines des ânes \\
\hline Sla7 le-n-ndar/Saleh Le-n-dar & Qui corrige la vision \\
\hline Khobz el-3a9rab & Pain du scorpion \\
\hline 3attasa & Celle qui fait éternuer
\end{tabular}

Les noms chargés de sens permettent de transmettre des connaissances, à l'ensemble de la population par un groupe souvent plus restreint possédant un savoir environnemental plus approfondi. C'est une démarche courante dans le cas de populations à transmission orale (Grenand 2002).

Par exemple ils permettent de distinguer Buplorum sp. et Euphorbia sp., relativement similaires morphologiquement parlant. Le second contient un latex (lib, le lait), à l'inverse du premier. Buplorum sp. n'a cependant jamais été regroupé, par aucun des informateurs sous le nom local de l'euphorbe, 7ullayba, «qui sécrète du lait »: «Ce n'est pas 7ullayba, c'est Khlechar " confirme un garçon. Cette capacité à différencier deux espèces très proches morphologiquement s'est observée aussi entre deux espèces d'Apiaceae: Ferula sp., Slilly, et Thapsia sp., Adries. Ces fleurs, jaunes et de hauteurs supérieures à un mètre sont très visibles dans les champs et largement répandues. Lors de l'exercice d'identification, les femmes d'âge mûr l'ont identifiée séparément : Adries "est plus épais que Slilly, et sa fleur est plus claire, et on n'en fait rien » nous a confirmé la mère de la famille en épluchant Slilly pour le manger cru en plein champ. À l'inverse, certaines espèces ne sont pas différenciées et sont regroupées sous un même nom. C'est le cas par exemple de Lewwaya, "celle qui s'enroule", qui rassemble l'ensemble des Campanulaceae, dont aucun usage n'a été répertorié au cours de nos enquêtes. Les noms peuvent être une manière de déceler des connaissances botaniques poussées, comme c'est le cas par exemple d'Erodium moschatum, « les cornes du mouton », pour lequel on nous explique qu'il fait référence à l'aspect du schizocarpe ${ }^{8}$, qui tourne, est éjecté et rentre dans le sol quand le fruit arrive à maturité. 


\section{Catégories d'usages et représentations culturelles}

51 Si l'on s'intéresse plus particulièrement aux plantes des deux catégories d'usages que nous avons mentionnées (Beqqula et alimentation animale), celles relatives à l'alimentation animale suivent le même schéma de catégorisation que les autres plantes en général (Fig. 9). $40 \%$ des noms des plantes relatifs à cet usage sont motivées. $61 \%{ }^{9}$ des noms font référence à une analogie de l'apparence de la plante avec un animal, $38 \%$ désignent quant à eux une fonction relative à l'usage de ces plantes par ou pour l'animal (alimentation). Par exemple, seules les chèvres mangent l'asperge sauvage Chokk l-m3iz, "les épines des chèvres ", Asparagus sp. Les préférences de l'âne sont aussi connues : les Asteraceae tubuliflores en particulier les chardons tel que le chardon marie sont nommées Chokk l-7mir «les épines des ânes ", que "l'âne mange, c'est sucré » (femme d'Aouiina Malha). Puis un faible pourcentage fait référence à une comparaison de l'apparence de la plante à un objet domestique, en analogie à l'apparence de la plante, ou à un évènement personnel.

Figure 9 : Pourcentage des différentes catégories de motivation des plantes utilisées pour l'alimentation animale

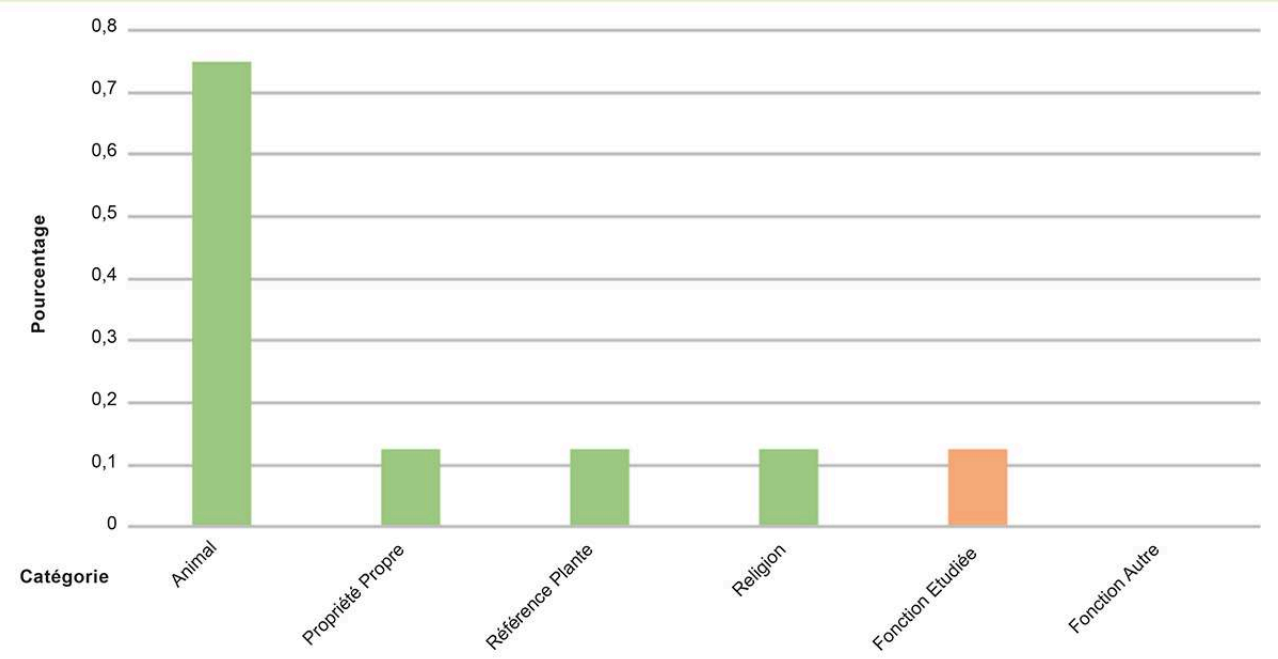

Celles de la Beqqula montrent que $35 \%$ des noms des plantes relatives à cet usage sont motivés, et ne font aucunement référence à sa fonction sensu stricto (Figure 10). Une seulement, bien que cela reste ambigu avec son apparence, désigne la fonction gustative de la plante (Bou-7ammo fjel (fjel, le radis) Barbarea sp.). 
Figure 10 : Pourcentage des différentes catégories de motivation des plantes utilisées pour la Beqqula

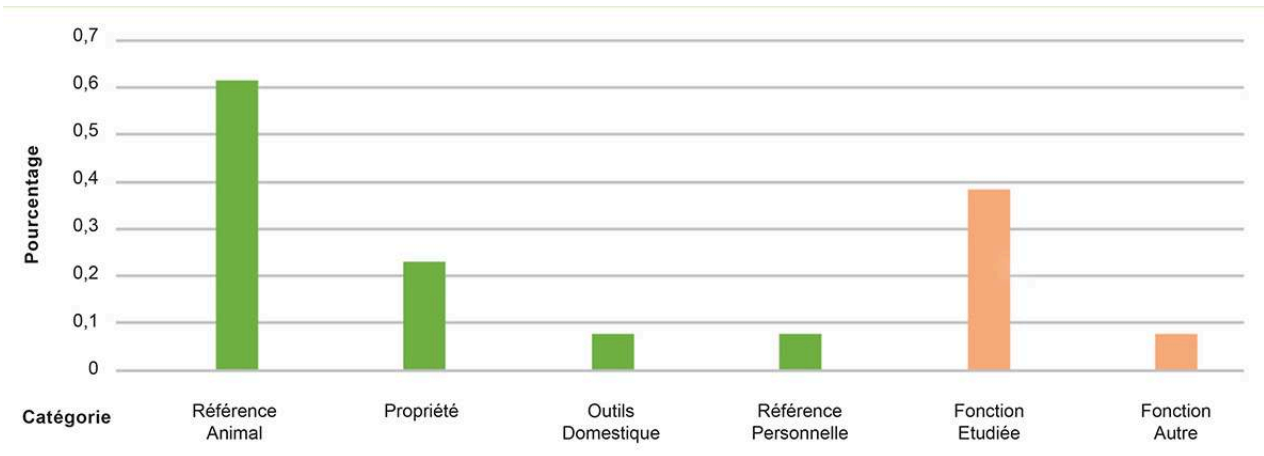

53 Dans cette catégorie, une fois encore, $75 \%$ fait référence à une analogie physique entre plante et animal. Un très faible pourcentage concerne des références à une caractéristique physique de la plante elle-même, à une autre plante, mais aussi à la religion.

Ainsi, ces connaissances fines signifiées par la composition de noms des herbes sauvages permettent de confirmer, en plus des usages, des représentations que les populations jbala de la zone d'étude entretiennent avec ces plantes sauvages. L'élément le plus marquant est que ces herbes font référence de façon majoritaire à des animaux sauvages ou domestiques, tout autant à des analogies avec des parties du corps des animaux (pieds, etc.), qu'aux préférences de ceux-ci, à savoir, ce qu'ils mangent. Ces noms ont été fabriqués au fil du temps selon des principes très complexes. Ainsi, par exemple, pour un certain plantain, Plantago sp., que l'on nomme Khobz el aqrab, "pain du scorpion ", on nous raconte que ce sont les petits bergers qui, en s'asseyant sur des rochers pour garder les animaux, qui utilisent la fleur du plantain longuement pédonculée, qu'ils tremperaient de leur salive et introduiraient dans des trous de rocher (en tenant le pédoncule) pour accrocher et extraire d'éventuels scorpion, ceci afin d'éviter de se faire piquer. Est-ce une fable ou une réalité ? Nous ne le saurons pas, mais cette histoire nous révèle l'immense complexité de ces façons de nommer les plantes ou des représentations symbolique qui président au fait de nommer.

Le fait qu'ils fassent référence au monde animal de façon aussi prégnante, montre en outre que plantes et animaux sont liés selon des modalités d'interactions tout autant symboliques (analogiques) que pragmatiques (ce que ces derniers mangeraient, les pains de...). Si l'on compare aux plantes cultivées, nous savons que celles-ci sont nommées sans ambiguïté aucune, sans se référer au monde animal. Serait-ce parce qu'elles sont sous le des plantes domestiquées, donc appartenant au monde des hommes? Ainsi une tomate est matecha, une olive zeytouna, une figue kermos etc. Cette abondance de référence au monde animal pour les herbes sauvages, pourrait aussi se référer au fait que ces plantes appartiennent aussi à d'autres, ces autres étant ainsi là bien reconnus comme faisant bien partie de l'agroécosystème. Ainsi dans d'autres cultures comme nous l'avons par exemple expérimenté en Indonésie, la plante sauvage qui a un équivalent proche parmi les espèces cultivée, fait souvent référence à son utilisation par un animal sauvage ou un être surnaturel, intangible (e.g. Limau bunian, citron des esprits équivalent sauvage des citrons cultivés, de même Durian hantu, le durian du fantôme une Bombacaceae dont le fruit vide s'oppose à celui rempli et béni pour sa succulence en Asie du Sud-est (Aumeeruddy 1994). Concernant les variétés de 
figuiers, Hmimsa et al. (2012) nous dit que la façon de nommer les variétés font référence à quelques animaux (la colombe, l'âne), mais plutôt du monde domestique.

Les noms de plantes adventices font peu référence au monde de l'homme (histoire personnelle, outils domestiques, etc.) hormis par l'intermédiaire d'un animal parfois domestique, voire d'une pratique humaine impliquant l'animal. Cela nous fournit les premiers éléments de perception de ces plantes sauvages, celles liées à l'utilisation pour l'alimentation animale en particulier. C'est à travers l'usage de ces plantes et la relation que l'homme entretien lui-même avec l'animal, dans ce lien affectif du nourrissage que se mettent en place les connaissances de ce que préfèrent ces animaux. Or, en plus de ce lien affectif du nourrissage, c'est une réelle relation communicative et interactionnelle que nous observons entre l'homme et l'animal, une relation qui passe par le souci du bien-être et de l'alimentation des bêtes. Cela se lit aussi dans le panel de connaissances lié aux plantes fourragères, allant par ailleurs bien au-delà de celles décrite par la motivation des noms, et par la forte place accordée à ces plantes au sein même des cultures de céréales par exemple.

Pour ce qui est de la Beqqula, les usages de cette dernière sont largement évocateurs. Ce plat est préparé par la majorité des foyers, et si des justifications économiques peuvent être mises en avant, elles passent après celles purement gustatives voire identitaires. Les plantes sont vendues au souk en saison et la préparation est très appréciée au point qu'on la trouve même dans des restaurants à Fès, qu'on y observe des pratiques de cueillette dans certaines friches urbaines et qu'elle est impliqué dans les réseaux d'échange et de dons.

\section{Conclusion}

Nous possédons ainsi de nombreux éléments gravitant autour des plantes sauvages, et du territoire concerné. Les différents ensembles de plantes, répartis de manière spécifique dans la mosaïque paysagère, possèdent des caractéristiques écologiques propres, et sont associées à des savoirs et savoir-faire adaptés, et éclairent sur les perceptions locales de la complexité écologique de l'agroécosystème. La relation fine à l'environnement, par la connaissance profonde de nombreux usages et caractéristiques des plantes sauvages, et l'identification de lieux épars de récolte par contact quotidien ont été mises en évidence. C'est au sein de l'espace vécu que se met en place cette connaissance, ce qui conforte les méthodes de travail adoptées. C'est également dans ce contexte que l'animal détient une place importante, largement mobilisée dans la nomenclature lorsqu'il s'agit de plantes. Ces interactions complexes entre monde domestique humain (la maison où est gardée la vache et son veau), les champs, les herbes sauvages, et l'ensemble des animaux sauvages qui habitent ces espaces nous permettent en outre de considérer qu'il n'y a pas une barrière stricte et infranchissable entre le domestiqué et le cultivé, comme il a été évoqué concernant les travaux sur le figuier et l'olivier (Aumeeruddy-Thomas et al. 2014, Hmimsa et al. ce volume). Ces ensembles que nous appelons sauvages et domestiques font partie d'un même collectif où divers niveaux d'interactions, divers types de liens, lient les humains et les nonhumains. La composition de ces mondes ruraux est ainsi plus complexe que les catégories agricoles standard, telle que les « mauvaises herbes » ou la distinction stricte entre cultivé et sauvage qui dissocient les humains des non-humains (plantes et animaux non-utiles). 
59 Des travaux similaires au Maroc montrent l'importance des connaissances ethnobotaniques et ethnozoologiques et leurs liens socio-historiques avec les territoires et l'espace vécu (Simenel 2007, Hmimsa et al. 2012, Delplancke \& Aumeeruddy-Thomas ce volume). Ces connaissances et ces liens aux territoires contribuent à l'identité culturelle des groupes sociaux marocains mais sont rarement reconnus en tant que tel, bien qu'ils participent à des fonctions importantes que sont la sécurité alimentaire et la capacité de faire face à des risques (Thiébault \& AumeeruddyThomas 2016, Michon et al. 2017).

60 Et, si la relation au territoire implique tant une relation à l'environnement qu'aux individus, nous avons pu mettre en lumière différentes composantes sociales gravitant autour de ces plantes sauvages. Il s'agit d'un savoir et savoir-faire avant tout féminin. On observe diverses modalités de savoirs parmi les habitants d'un même lieu et entre les lieux ainsi que divers modes de transmission des savoirs variant selon ces lieux et l'hétérogénéité des usages. Ces savoirs se transmettent comme l'ont évoqué Powell et al. (2014) au-delà des lieux de cueillette, possiblement via les souks, même si nous avons vu que les savoirs des souks sont moins riches. Ces savoirs ne peuvent dès lors pas se transmettre essentiellement par les marchés. Et, si l'on observe une érosion des connaissances relatives aux plantes par les nouvelles générations, cela illustre une évolution du rapport de ces populations à leur environnement, qu'il s'agirait d'approfondir.

61 Enfin, ce travail n'a permis que de réaffirmer l'intérêt d'une approche interdisciplinaire, entre l'ethnobotanique, l'écologie et la nécessité de développer la linguistique comme porte d'entrée pour comprendre la relation qui unit une société à son environnement, en complémentarité avec l'étude des pratiques, des usages et des représentations des plantes spontanées.

\section{Remerciements}

Nous souhaitons remercier particulièrement Fouzia El Ghazaz, sa famille et belle-famille pour leur aide précieuse apportée lors du travail de terrain. Merci également à Joël Mathez pour ses conseils botaniques. Nous remercions Dominique Caubet et Younes Hmimsa qui ont contribué à la mise en forme des terminologies vernaculaires dans cet article. Ce travail a été possible grâce au soutien du Projet International de Coopération Scientifique (PICS) France-Maroc (CNRSCNRST) intitulé "La montagne et ses savoirs ", ainsi qu'au GDR Mosaïque 3353 (Agroécosystème, Agrobiodiversité et Environnement. Domestication et Innovations). Enfin, le stage de Master 1 effectué a été accueilli par le CEFE UMR 5175 au sein de l'Équipe Interactions Bioculturelles que nous remercions également.

\section{BIBLIOGRAPHIE}

Ater M. \& Hmimsa Y. 2013 - Agrodiversité des agroécosystèmes traditionnels du pays Jbala (Rif, Maroc) et produits de terroirs. In : Ilbert H., Tekelioglu Y., Çagatay S. \& Tozanli S. (Ed.) Indications Géographiques, dynamiques socio-économiques et patrimoine bio-culturel en Turquie et dans les pays 
méditerranéens. Montpellier : CIHEAM, (Options Méditerranéennes : Série A. Séminaires Méditerranéens ; 104) : 197-208.

Aumeeruddy Y. 1994 - Représentations et gestion paysannes des agroforêts en périphérie du Parc National Kerinci Seblat à Sumatra, Indonésie. People and Plants Working Paper Series, No 3, Paris, UNESCO, (versions anglaise et espagnole disponibles) 46 p. http://www.peopleandplants.org/ storage/working-papers/wp3sp.pdf

Aumeeruddy-Thomas Y., Hmimsa Y., Ater M. \& Khadari B. 2014 - Beyond the divide between wild and domesticated: spatiality, domesticity and practices pertaining to fig (Ficus carica L.) and olive (Olea europaea L.) agroecosystems in Morocco. In : Chevalier A., Marinova E., Peña-Chocarro L. (Ed.) Crops and people: choices and diversity through time. Brussels, Earth EU, London, OXFAM : 191-197.

Aumeeruddy-Thomas Y., Moukhli A., Haouane H. \& Khadari B. 2017 - Ongoing domestication and diversification in grafted olive-oleaster agroecosystems in Northern Morocco. Regional Environmental Change 17 : 1315-1328. DOI 10.1007/s10113-017-1143-3

Bellakhdar J. 1978 - Médecine traditionnelle et toxicologie Ouest-sahariennes : Contribution à l'étude de la pharmacopée marocaine. Rabat, Techniques Nord-Africaines, 357 p.

Bellakhdar J. 1997 - La pharmacopée marocaine traditionnelle : médecine arabe ancienne et savoir populaires. Paris, Ibis Presse, 760 p.

Blanc-Pamard C. 1986 - Dialoguer avec le paysage ou comment l'espace écologique est vu et pratiqué par les communautés rurales des hautes terres malgaches. In : Chatelin Y. \& Riou G. (Ed.) Milieux et paysages : essai sur diverses modalités de connaissance. Paris, Masson : 17-36.

Bouby L. 2000 - Production et consommation végétales au Bronze final dans les sites littoraux languedociens. Bulletin de la Société Préhistorique de France 97 (4) : 583-594.

Colombel V. \& Tersis N. (Ed.) 2002 - Weeds and domesticates: Evolution in the man-made habitat. Economic Botany 29 (2) : 99-107.

Friedberg C. 1986 - Classifications populaires des plantes et modes de connaissances. In : Tassy P. (Ed.) L'ordre et la diversité du vivant. Paris, Fondation Diderot, Fayard : 23-49.

Garine-Wichatitsky E. 1997 - Sauvage ou domestique ? Remarques sur l'inventaire des plantes à brèdes chez les Gimbe et les Duupa du Nord-Cameroun. In : Barreteau D., Dognin R. \& von Graffenrie C. L'homme et le milieu végétal dans le bassin du lac Tchad : 311-326.

Grenand F. 2002 - Stratégies de nomination des plantes cultivées dans une société tupi-guarani, les Wayãpi. Amerindia, 26/27 : 209-247.

Hmimsa, Y. \& Ater M. 2008 - Agrodiversity in the traditional agrosystems of the Rif mountains (North of Morocco). Biodiversity 9 (1-2) : 78-81.

Hmimsa Y., Aumeeruddy-Thomas Y. \& Ater M. 2012 - Vernacular taxonomy, classification and varietal diversity of fig (Ficus carica L.) among jbala cultivators in Northern Morocco. Human Ecology $40: 301-313$

Leonti M, Nebel S., Rivera D. \& Heinrich M. 2006 - Wild Gathered Food Plants in the European Mediterranean . A Comparative Analysis. Economic Botany 60 (2) : 130-142.

Lins Neto E., Peroni N., Casas A., Parra F., Aguirre X., Guillén S. \& Albuquerque U. 2014 - Brazilian and Mexican experiences in the study of incipient domestication. Journal of Ethnobiology and Ethnomedicine 10 (1) : 33. https://doi.org/10.1186/1746-4269-10-33 
Martin G. 1995 - Ethnobotany. A People and Plants Conservation Manual. London, Chapman and Hall, 268 p.

Médail F.\& Quézel P. 1999 - Biodiversity Hotspots in the Mediterranean Basin: Setting Global Conservation Priorities. Conservation Biology 13 (6) : 1510-1513.

Michon G., Berriane M., Romagny B. \& Alifriqui M. 2017 - Les savoirs locaux peuvent-ils inspirer des solutions adaptatives dans les arrière-pays du Maroc ? Hespéris-Tamuda LII (1) : 319-356.

Nassif F. \& Tanji A. 2013 - Gathered food plants in Morocco: the long forgotten species in ethnobotanical research. Life sciences leaflets 3 (3) : 17-54.

Pennec F., Wencelius J., Garine E., Raimond C. \& Bohbot H. 2012 - Flame v1.1 - Free-List Analysis Under Microsoft Excel. Paris, CNRS. [En ligne] http://www.mae.u-paris10.fr/lesc/spip.php? article63

Petit S., Thenail C., Chauvel B., Le Cœur D. \& Baudry J. 2008 - Les apports de l'écologie du paysage pour comprendre la dynamique de la flore adventice. Innovations Agronomiques $3: 49-60$.

Powell B., Ouarghidi A., Johns T., Ibn Tattou M. \& Eyzaguirre P. 2014 - Wild leafy vegetable use and knowledge across multiple sites in Morocco: a case study for transmission of local knowledge? Journal of Ethnobiology and Ethnomedicine $10: 34$.

Rosenberger B. 1980 - Cultures complémentaires et nourritures de substitution au Maroc (XVeXVIIIe siècle). Annales. Économies, Sociétés, Civilisations 35 (3) : 477-503. [En ligne] http:// www.persee.fr/web/revues/home/prescript/article/ahess_03952649_1980_num_35_3_282648. [Consulté le 20 juin 2017].

Simenel R. 2007 - L'origine est aux frontières : espace, histoire et société dans une terre d'exil du sud marocain. Doctoral dissertation, Paris 10,397 p.

Tanji A. \& Nassif F. 1995 - Edible weeds in Morocco. Weed Technology 9 : 617-620.

Thiébault S. \& Aumeeruddy-Thomas Y. 2016 - Local knowledge, scientific knowledge and food security. Introduction. In : Thiébault S. \& Moatti J.-P. (Ed.) The Mediterranean Region Under Climate Change. A Scientific Update. Marseille, IRD Editions : 519-522. http://fr.calameo.com/read/ 00331938471

Troin J.F. 1975 - Les souks marocains : marchés ruraux et organisation de l'espace dans la moitié nord du Maroc. Aix-en-Provence, Edisud, I : 502 p. ; II : 28 pl.

Willcox G. 2012 - Searching for the origins of arable weeds in the Near East. Vegetation History and Archaeobotany. 21 (2) : 163-167

\section{ANNEXES}

Liste des noms de plantes répertoriées 


\begin{tabular}{|c|c|c|c|c|}
\hline $\mathrm{N}^{\circ}$ & Noms vernaculaires & Espèce $/ \mathbb{N}^{\circ}$ Herbier & Famille & Signification \\
\hline 1 & 2arn el Kebch & Erodium sp/ LCYAT 80 & Geraniceae & Les cornes du mouton \\
\hline 2 & 3attasa & LCYAT 137 & Asteraceae & Qui fait éternuer \\
\hline 3 & 3icha swalefl/soalef & & & Les longues couettes de cheveux de Aichai \\
\hline 4 & 3ugiff & Scorpiorus muricatus/LCYAT 86 & Fabaceae & \\
\hline 5 & 7enna d-el-ba2ra & LCYAT 95 & Asteraceae & Hénné de la vache \\
\hline 6 & 7ommayda & Rumex sp/LCYAT 73 & Polygonaceae & \\
\hline 7 & 7orray9a & & & \\
\hline 8 & 7orrey9a melsa & Mercurialis annua/LCYAT129 & Euphorbiaceae & \\
\hline 9 & 7orricha I-senklard & LCYAT 2 & Boraginaceae & Rugeux \\
\hline 10 & 7ulba & & & \\
\hline 11 & 7ullayba & Euphorbia helioscopa/LCYAT 14 LCYAT 113 & Euphorbiaceae & Laiteuse \\
\hline 12 & 92anaria/Khorchouf & & Asteraceae & \\
\hline 13 & 9ossat 1'7ayya & $\begin{array}{l}\text { Erythrae centaurium/Centaurea sp/Valerianel- } \\
\text { la sp LCYAT } 4 \text { LCYAT } 112 \text { LCYAT } 122\end{array}$ & $\begin{array}{l}\text { Cencianaceae } \\
\text { Asteraceae } \\
\text { Valerianaceae } \\
\end{array}$ & \\
\hline 14 & A'Ddad & & Asteraceae & \\
\hline 15 & Adries & Thaspia (garganica)/LCYAT 37 & Apiaceae & \\
\hline 16 & Asra/Zamij & & Asteraceae & \\
\hline 17 & Azir & & Lamiaceae & \\
\hline 18 & Babonj & Ormenis sp/LCYAT 85 & Asteraceae & \\
\hline 19 & Baboung/N-nowwara lbayda & & Asteraceae & La fleur blanche \\
\hline 20 & Bagraman & & & \\
\hline 21 & Beha safi & & & \\
\hline 22 & Beqqul d-el-7orr & & Asteraceae & \\
\hline 23 & Beqqul L-7orrin & & Asteraceae & \\
\hline 24 & Berwag & & Liliaceae & \\
\hline 25 & bezzol L-3awada & LCYAT 84 & & Le sein de jument \\
\hline 26 & Bibbek & & Apiaceae & \\
\hline 27 & Boseyla d-dib & & Liliaceae & Le petit oignon loup/chacal \\
\hline 28 & Boseyla/Griba d S3ay & LCYAT 108 & Liliaceae & Gri b = jarre du plaidoyé \\
\hline 29 & Bou-3oqda & LCYAT 81 & Asteraceae & Celui avec un nœeud \\
\hline 30 & Bou-7amdouna & Echium paviflorum/LCYAT 114 & Boraginaceae & \\
\hline
\end{tabular}

\begin{tabular}{l|l|l|l|l}
\hline$N^{\circ}$ & Noms vernaculaires & Espèce/N ${ }^{\circ}$ Herbier & Famille & Signification \\
\hline $\mathbf{3 1}$ & Bou-7ammo & Barbarea sp & Brassicaceae & \\
\hline $\mathbf{3 2}$ & Bou-7ammo fjel & Barbarea sp/LCYAT 78 & Brassicaceae & Fjeul = Radis \\
\hline $\mathbf{3 3}$ & Bou-7hamo cherriat & Barbarea sp/LCYAT 56 & Brassicaceae & \\
\hline $\mathbf{3 4}$ & Bou-bel/ boubal & Ferula sp/LCYAT 124 & Apiaceae & \\
\hline $\mathbf{3 5}$ & Bou-chnikh & Ammi (visnaga)/LCYAT 27 & Apiaceae & \\
\hline $\mathbf{3 6}$ & Bou-le3mane/Bula3mane & Papaver rhoeas/LCYAT 80 & Papaveraceae & \\
\hline $\mathbf{3 7}$ & Bou-swifa & Anchyala (integrifolia)/LCYAT 111 & Asteraceae & Celui avec la laine \\
\hline $\mathbf{3 8}$ & Bou-Zwiwel & & Asteraceae & \\
\hline $\mathbf{3 9}$ & Chafa Tammo & & & Lèvres de Tammo \\
\hline $\mathbf{4 0}$ & Cherriat & Barbarea sp & Brassicaceae & \\
\hline $\mathbf{4 1}$ & Chiba & Artemisia absinthium/LCYAT 44 & Asteraceae & \\
\hline $\mathbf{4 2}$ & Chmendar & & & \\
\hline $\mathbf{4 3}$ & Chokk I-7mir & Silybum marianum/Carlina sp/Galactites/LCY- & Asteraceae & Epine l'ane \\
\hline $\mathbf{4 4}$ & Chokk I-m3iz & & & Les épines des chèvres \\
\hline $\mathbf{4 5}$ & Chokka & & Asteraceae & \\
\hline $\mathbf{4 6}$ & Chowwal L-Khrof & Orobanche sp/LCYAT 133 & Orobanchaceae & Queue du mouton \\
\hline $\mathbf{4 7}$ & Chtaba & & & Balais \\
\hline $\mathbf{4 8}$ & Dawma & & & \\
\hline $\mathbf{4 9}$ & Dlem & & & \\
\hline $\mathbf{5 0}$ & Dro & Pistacia lentiscus & Fabaceae & Comme la jarre \\
\hline $\mathbf{5 1}$ & El-7orf & LCYAT 88 & Asteraceae & \\
\hline $\mathbf{5 2}$ & Fassa & Medicago sp & Asteraceae & \\
\hline $\mathbf{5 3}$ & Ferrona & & & \\
\hline $\mathbf{5 4}$ & Fjeul/jeujer & Barbarea sp/LCYAT 131 LCYAT 87 & Brassicaceae & \\
\hline $\mathbf{5 5}$ & Fliyo & Mentha pulegium/LCYAT 101 & & \\
\hline $\mathbf{5 6}$ & Fowwa7a & & & \\
\hline $\mathbf{5 7}$ & Fowwa7a/Bou-3aej n7al & Centaurea sp/LCYAT 16 & & \\
\hline $\mathbf{5 8}$ & Gouriba d-s-S3ay & Tripodion (tetraphyllum)/ LCYAT 108 & \\
\hline $\mathbf{5 9}$ & Guernin & & & \\
\hline $\mathbf{6 0}$ & Guernina & & & \\
\hline & & & & \\
\hline
\end{tabular}




\begin{tabular}{|c|c|c|c|c|}
\hline $\mathrm{N}^{\circ}$ & Noms vernaculaires & Espèce $/ \mathbb{N}^{\circ}$ Herbier & Famille & Signification \\
\hline 61 & Isla & Bromus sp/Brachypodium sp/LCYAT 97 & Poaceae & \\
\hline 62 & Jlibna & Vicia sp/LCYAT 132 LCYAT 139 & Fabaceae & \\
\hline 63 & Kersanna & Vicia sativa & Fabaceae & \\
\hline 64 & Khlechar & Buplorum sp & euphorbiaceae & \\
\hline 65 & $\begin{array}{l}\text { Khobbiza/ ba229ula (baqqu- } \\
\text { la) }\end{array}$ & Malva sp/LCYAT 72 & Malvaceae & \\
\hline 66 & Khobz el-3a9rab & Plantago sp/LCYAT 9 & Plantaginaceae & Pain du scorpion \\
\hline 67 & Khobz el-ghrab & LCYAT 119 & Asteraceae & Pain des corneilles/corbeaux \\
\hline 68 & Khortal & Avena (sativa)/LCYAT 34 & Poaceae & \\
\hline 69 & Khrawia & LCYAT 17 & Poaceae & \\
\hline 70 & Ko77ayla & LCYAT 138 & & La noiraude \\
\hline 71 & Kra3 djaja & & Asteraceae & Pied de la poule \\
\hline 72 & Kreye3 fellus/ Rijyyel fellus & & Asteraceae & Petit pied du poussin \\
\hline 73 & L'7archa machi & & & \\
\hline 74 & Le7yat I-3atros & Tragopogon sp/LCYAT 102 & Asteraceae & La barbe du bouc \\
\hline 75 & Lewwaya & $\begin{array}{l}\text { Campanula sp/Convolvulus sp/LCYAT } 118 \\
\text { LCYAT } 36\end{array}$ & Campanulaceae & Celle qui grimpe \\
\hline 76 & Le-zzaz & & & \\
\hline 77 & Lianou & Lolium sp/LCYAT 19 & Poaceae & \\
\hline 78 & L-losse9a & Rubia sp/LCYAT 11 LCYAT 30 LCYAT 45 & Rubiaceae & La petite collante \\
\hline 79 & L-mrara & Cichorum sp/LCYAT 96 & Asteraceae & La petite amère \\
\hline 80 & Matecha d'17aya & Solanum sp/LCYAT 99 & Solanaceae & La tomate du serpent \\
\hline 81 & Menta & Menta sp & Lamiaceae & \\
\hline 82 & Merdeddoch & & & \\
\hline 83 & Merriwa & Marrubium (vulgare)/LCYAT 53 & Labiae & \\
\hline 84 & Misla & & & \\
\hline 85 & Mkhinza & & & La petite puante \\
\hline 86 & Mochayta & & & Fleur ressemble à un peigne \\
\hline 87 & Mollisa & LCYAT 136 & & Très lisse (la petite lisse) \\
\hline 88 & Mrinsla/chettaba melsa & Sixalix atropurpurea/LCYAT 106 & Caprifoliaceae & Balais lisse \\
\hline 89 & $\mathrm{Na3na3/liqama}$ & & & \\
\hline 90 & Nefla & Trifolium sp/LCYAT 134 & Fabaceae & \\
\hline
\end{tabular}

\begin{tabular}{|c|c|c|c|c|}
\hline $\mathbf{N}^{\circ}$ & Noms vernaculaires & Espèce $/ \mathrm{N}^{\circ}$ Herbier & Famille & Signification \\
\hline 91 & Nejm & Brachipodium sp/LCYAT 93 & Poaceae & \\
\hline 92 & Nowwar d ch'chahd & & Asteraceae & \\
\hline 93 & Nowwar d j-jemra & & Asteraceae & Fleur de la braise/étincelle \\
\hline 94 & Nowwar I-jenna & & Asteraceae & Fleur du paradis \\
\hline 95 & Nowwara sefra & & Asteraceae & Fleur jaune \\
\hline 96 & Qinqer & Misopates sp/LCYAT 92 & Scrophulariaceae & La frange du serpent \\
\hline 97 & Qrizza & Anagalis sp/LCYAT 105 & Primulaceae & La frange du serpent \\
\hline 98 & Rbi3a o safi & & Poaceae & \\
\hline 99 & Rejla & & & Pied \\
\hline 100 & Sanaria/Kanaria & & Asteraceae & \\
\hline 101 & Sannuj & & Renonculaceae & \\
\hline 102 & Selk & & & \\
\hline 103 & $\begin{array}{l}\text { Sla7 le-n-ndar/ Saleh Le-n- } \\
\text { dar }\end{array}$ & LCYAT 43 & & Qui corrige la vision \\
\hline 104 & Slilly & Ferula sp/LCYAT124 & Apiaceae & \\
\hline 105 & Sqaliya & Triticum monococcum & Poaceae & \\
\hline 106 & Ta3ora & LCYAT 5 & Asteraceae & \\
\hline 107 & Taymante & & & \\
\hline 108 & Tisa & & & \\
\hline 109 & Traylan & Amimagus sp/LCYAT 74 & Apiaceae & \\
\hline 110 & Tsa3ora & & Asteraceae & \\
\hline 111 & T-tuma d-dib & & & Ail du loup \\
\hline 112 & Yierna & & & \\
\hline 113 & Z3etra & Lavendula multifidia/LCYAT 29 & Labiae & Petite Za3ter \\
\hline 114 & Za3tar & LCYAT 31 & & \\
\hline 115 & Zarnij & & Asteraceae & \\
\hline 116 & Ziayyta & & Apiaceae & Huileuse \\
\hline 117 & Zwan & Phalaris sp/LCYAT 17 & Poaceae & \\
\hline 118 & Z-ztema de ddib & Eragium sp/LCYAT 103 & & \\
\hline
\end{tabular}

\section{NOTES}

1. L'Ihyâ (Le Renouveau des Sciences religieuses) est un traité d'éthique musulman, portant sur les connaissances et devoirs de l'Homme envers Dieu (cinq piliers de l'Islam), le savoir-vivre, les 
maux et les vertus morales. Il est l'œuvre majeure du grand érudit sunnite Abu-Hamid Al-Ghazali (Algazel en latin) (1058-1111). (Source: Ralph Stehly, Professeur d'histoire des religions, Université de Strasbourg; Bibliothèque numérique mondiale, Library of Congress: https:// www.wdl.org/fr/item/7457/)

2. Concernant les modes de transcription des termes arabes, voir Aumeeruddy-Thomas et al. ce numéro.

3. «Groupement d'habitations, fixe ou mobile, temporaire ou permanent, réunissant des individus liés par une parenté fondée sur une ascendance commune en ligne paternelle; Division administrative de base, en Afrique du Nord » (source : http://www.cnrtl.fr/lexicographie/Douar)

4. Citations issues d'entretiens réalisés en français, ou arabe marocain, grâce à l'aide de Fouzia El Ghazaz (traduction comprise).

5. Cette liste comprend l'ensemble des noms évoqués par les informateurs, incluant des petites variations pour a priori une même espèce. Le travail d'identification botanique n'a pas été abouti (Tableau 1) et les noms relevés ici sont l'œuvre d'un seul travail de terrain réalisé lors du stage M1 de Louise Clochey. La richesse de notre travail repose avant tout sur une analyse qualitative.

6. Identification de l'échantillon herborisé, herbier réalisé au cours du stage, et déposé à l'Institution Botanique de Montpellier.

7. L'ensemble des pourcentages suivants reste relatif compte tenu du point exposé en note 2 . Ils sont fournis à titre indicatif. La richesse de notre travail repose avant tout sur une analyse qualitative.

8. Fruit sec ou rarement charnu initialement indéhiscent.

9. Les items pouvant se trouver dans deux catégories, lorsque les enquêtes qualitatives révèlent des affiliations multiples.

\section{RÉSUMÉS}

L'agriculture, pilier économique principal de la région du Rif (Nord du Maroc), reste sous sa forme de système agro-sylvo-pastoral, avant tout destinée à l'autoconsommation. Ce système traditionnel entraîne la présence d'une mosaïque de milieux agraires et forestiers, riches d'une forte agrobiodiversité. L'objet de cette contribution est de présenter les pratiques, usages et représentations liés aux plantes adventices des cultures dans cette région - Ain Mediouna, province de Taounate plus précisément. Indissociables de l'ensemble de la flore spontanée de l'agroécosystème, c'est en considérant cette dernière dans sa globalité que nous avons pu apporter des réponses à nos questionnements. Nos travaux s'appuient ainsi sur une démarche ethnobotanique, incluant des relevés botaniques et des enquêtes ciblées sur l'usage des plantes spontanées de l'agroécosystème, couplée à une approche ethnographique de terrain d'observation participante, incluant des entretiens ouverts et le suivi prolongé des activités et des relations des habitants entre eux. Nous abordons à travers des corpus recueillis auprès de femmes et d'hommes de tous âges, ainsi que d'enfants, la façon dont les connaissances des plantes adventices, et plus largement des plantes spontanées, interviennent dans leur vie quotidienne, au travers des activités diverses qu'elles engendrent, ainsi que leurs liens à l'espace agraire et à autrui. Nous illustrons notre propos en montrant l'ensemble des connaissances qui gravitent autour de : (1) la préparation d'un plat cuisiné à base de légumes-feuilles sauvages, la beqqula, (2) le nourrissage de jeunes veaux avec les plantes printanières spontanées. Les taxonomies vernaculaires, en particulier les façons de nommer ces plantes spontanées, ont été 
analysées. Elles renvoient notamment à des références au monde naturel très distinctes de la façon de nommer les plantes cultivées. Nous mettons en évidence également comment les connaissances et les usages varient selon les âges des habitants et en fonction des activités spécifiques (alimentation animale, activités pastorales, fabrication de plats à usage alimentaire, etc.). Ces deux cas (beqqula et nourrissage des animaux) permettent alors d'aborder la place des plantes spontanées et plus précisément des plantes adventices des cultures dans l'alimentation des hommes, d'une part, et des animaux, d'autre part, et de proposer ainsi, à travers l'analyse des pratiques qui y sont liées, une ébauche du rôle de ces plantes sur les relations entre les hommes, et entre les hommes et les animaux dans le cadre du territoire étudié.

Agriculture, the main economic pillar of the Rif area (Northern Morocco), has remained in the form of an agro-sylvo-pastoral system first and foremost intended for local consumption. This traditional system brings about a patchwork of farming and forestry environments with a very high agro-biodiversity. The purpose of this paper is to show the practices, habits and perceptions related to weeds in agricultural fields in this region -more specifically Ain Mediouna, in Taounate province. Weeds are inseparable from the whole spontaneous flora and it is only by considering the latter in its entirety that our questions might be answered. In fact, an ethnobotanical approach -involving botanical surveys and targeted investigations into the use of spontaneous plants of the agro-ecosystem combined with ethnographic field methods including participant observation and open interviews as well as the extended follow-up of the inhabitants' activities and relations among themselves - underpins our work. Through corpora collected from elderly and younger women and men as well as children, we analyze the way their knowledge on weeds and more widely spontaneous plants influences their daily lives and various activities, their ties with the agricultural space and with other people. To illustrate our work, we show the whole body of knowledge revolving around (1) cooking a dish of wild leafy vegetables called beqqula and (2) feeding young calves with spontaneous spring plants. Vernacular taxonomies, and more specifically the ways of naming these spontaneous plants, were analyzed. It is to be noted that the way they refer to the natural world is very different from the way cultivated plants are named. How knowledge and practices vary with the inhabitants' ages and according to specific activities (animal feeding, pastoral pursuits, making food dishes etc.) is also highlighted. Thus the role of spontaneous plants and more precisely of weeds in feeding humans on the one hand, and animals on the other hand, can be dealt with through both cases (beqqula and animal feeding). Moreover, analyzing the practices linked to them, allows an outline of the role played by these plants in the relations between people, and among people and animals, within the investigated territory.

\section{INDEX}

Mots-clés : ethnobotanique, ethnologie, mosaïque agraire, plantes adventices, pratiques alimentaires, légumes-feuilles sauvages, fourrage, Jbala, Rif, nord du Maroc

Keywords : ethnobotany, ethnology, agricultural mosaic, adventitious plants, food practices, wild edible herbs, forage, Jbala, Rif, Northern Morocco.

\section{AUTEURS}

\section{LOUISE CLOCHEY}

Ethnobotanique, Étudiante - Université Paul-Valéry Montpellier 3 - Site Saint Charles - Rue du Professeur Henri Serre - 34080 Montpellier 


\section{YILDIZ AUMEERUDDY-THOMAS}

Ethnobotanique, Directrice de recherche CNRS - UMR 5175, Equipe Interactions Bioculturelles Centre d'Écologie Fonctionnelle et Évolutive (CEFE) - 119, route de Mende - 34293 Montpellier 